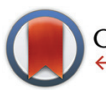

CrossMark click for updates

Cite this: Org. Biomol. Chem., 2016, 14,3963

Received 18th February 2016,

Accepted 29th March 2016

DOI: $10.1039 / \mathrm{c} 6 \mathrm{ob} 00388 \mathrm{e}$

www.rsc.org/obc

\title{
Metal-assisted addition of a nitrate anion to bis(oxy)enamines. A general approach to the synthesis of $\alpha$-nitroxy-oxime derivatives from nitronates $\uparrow$
}

\author{
Yana A. Naumovich, ${ }^{a}$ Victoria Emily Buckland, ${ }^{b}$ Dmitry A. Sen'ko, ${ }^{c}$ \\ Yulia V. Nelyubina, ${ }^{d}$ Yulia A. Khoroshutina, ${ }^{a}$ Alexey Yu. Sukhorukov*a and \\ Sema L. loffe ${ }^{a}$
}

\begin{abstract}
The synthesis of $\alpha$-nitroxy-substituted oxime derivatives has been achieved by an unprecedented metalassisted addition of a nitrate anion to bis(oxy)enamines, which are readily available from nitronates or nitroalkanes. The method has a broad scope and provides access to $\alpha$-nitroxy-oximes and their cyclic ethers including nitroxy-substituted isoxazolines and dihydro-1,2-oxazines, which are of interest as potential $\mathrm{NO}$-donors and intermediates in the synthesis of bioactive molecules.
\end{abstract}

\section{Introduction}

Nitrate esters are widely used in pharmaceuticals as efficient NO donors ${ }^{1}$ with vasodilative, ${ }^{1 a}$ vasorelaxant, ${ }^{1 b}$ and anti-tumor activities. ${ }^{1 c}$ Since NO is associated with the cytotoxic and antimicrobial functions of the immune system, the introduction of a nitroxy group in natural molecules and pharmaceuticals significantly enhances their efficacy. ${ }^{1 d}$ This strategy is widely applied in the design of hybrid drugs. ${ }^{1 d-h}$

In organic synthesis, nitrates are considered convenient substrates for nucleophilic substitution reactions, due to the good leaving group ability of the $\mathrm{ONO}_{2}$ fragment. ${ }^{2}$ Nitrates are employed as intermediates in the synthesis of alcohols ${ }^{3}$ and aldehydes $^{4}$ from the corresponding halides. Furthermore, nitrates of small alcohols are also well-known high-energy materials and components of explosive compositions. ${ }^{5}$

Traditional methods for the introduction of the $\mathrm{ONO}_{2}$ fragment in organic molecules are based either on the direct nitration of alcohols ${ }^{6}$ or on the nucleophilic $\mathrm{S}_{\mathrm{N}} 1$-type substitution of halides in haloalkanes, upon the action of silver or mercury nitrate. ${ }^{7}$ Another method substantially less explored

${ }^{a}$ N.D. Zelinsky Institute of Organic Chemistry, Leninsky prospect 47, 119991 Moscow, Russia.E-mail: sukhorukov@ioc.ac.ru; Fax: +7 499 1355328; Tel: +7 4991355329

${ }^{b}$ National Junior College, Hillcrest Road 37, 288913, Singapore

${ }^{c}$ Moscow Chemical Lyceum, Tamozhenniy proezd 4, 111033 Moscow, Russia

${ }^{d}$ A.N. Nesmeyanov Institute of Organoelement Compounds, Vavilov str. 28 ,

119991 Moscow, Russia

$\dagger$ Electronic supplementary information (ESI) available: NMR, IR and UV-Vis spectra for new compounds, X-ray data for 2g. CCDC 1419082. For ESI and crystallographic data in CIF or other electronic format see DOI: 10.1039/c6ob00388e is nucleophilic addition of a nitrate anion to double carboncarbon bonds. ${ }^{8,9}$ Such processes are usually realized under oxidative conditions (Scheme 1, eqn (1)), ${ }^{8}$ or initiated by the addition of an electrophile to a $\mathrm{C}=\mathrm{C}$ bond, followed by the nucleophilic attack of a nitrate anion on the carbocation or onium ion (Scheme 1, eqn (2)). ${ }^{9}$ However, examples of direct addition of a nitrate anion to an activated carbon-carbon double bond as well as its participation in $\mathrm{S}_{\mathrm{N}}{ }^{\prime}$ type substitution reactions are, to the best of our knowledge, unprecedented to date.

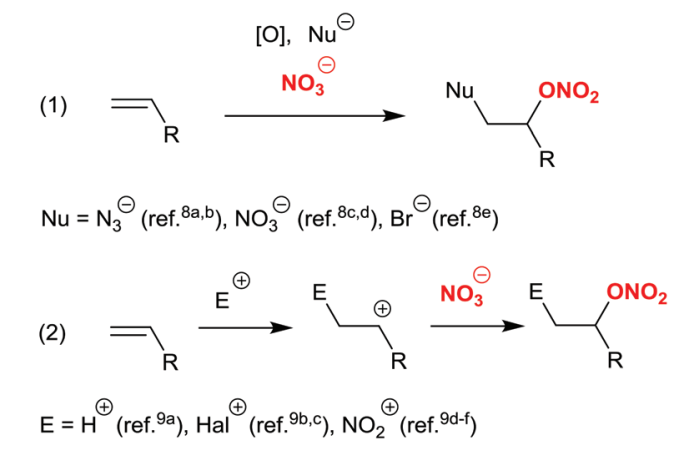

This work:

(3)

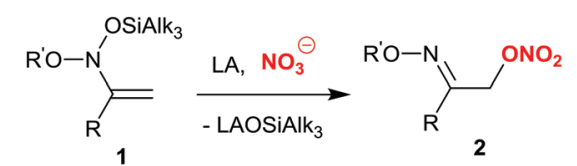

LA - Lewis acid

Scheme 1 Addition of a nitrate anion to a C,C-double bond. 
The carbon-carbon double bond in bis(oxy)enamines 1, readily available by silylation of nitronates, ${ }^{10}$ is easily attacked by some nucleophiles, thus forming $\alpha$-substituted oximes and their ethers as a result of a formal $S_{\mathrm{N}}{ }^{\prime}$ substitution of the silyloxy group. ${ }^{11}$ We were interested in employing nitrate anions as nucleophiles in reactions with bis(oxy)enamines, in order to obtain $\alpha$-nitroxy-oximes and their cyclic ethers (Scheme 1, eqn (3)). These products are virtually unknown in the literature, ${ }^{12}$ though first attempts to synthesize simple $\alpha$-nitroxy-oximes (so-called "nitrosates") by nitration of some olefins with $\mathrm{N}_{2} \mathrm{O}_{4}$ date back to the 19th century in the studies of Guthrie, ${ }^{13 a}$ Wallach ${ }^{13 b}$ and Ipatiew. ${ }^{13 c}$ However, in those times the structure of "nitrosates" could not be determined unambiguously.

\section{Results and discussion}

Recently, we have demonstrated that the reactions of bis(oxy) enamines with halide anions are promoted by Lewis acids, in particular transition metal and magnesium salts. ${ }^{14}$ Therefore, to achieve the desired transformation we studied the interactions of model cyclic bis(oxy)enamine 1a with a series of main and transition group metal nitrates (Table 1). In our experiments, crystal hydrates were usually used as they were the most readily available form of metal nitrates. The latter were dissolved in tetrahydrofuran (in the event of insolubility, dimethylformamide was used as a solvent instead) and treated with a solution of $\mathbf{1 a}$ in dichloromethane.

As can be seen from the data in Table 1, almost all studied metal nitrates produced the target product 2 a with full conversion of enamine 1a. An exception was lithium nitrate which gave low conversion of the starting material (Table 1, entry 1). The major by-products identified in experiments were 3-hydroxymethyl-substituted 1,2-oxazine 3a, its trimethylsilyl ether $\mathbf{4 a}$, as well as formate 5a and 1,4-butanediol ether nitrate 6a. Products $5 \mathbf{a}$ and $6 \mathbf{a}$ arise from competitive addition of solvent to bis(oxy)enamine 1a (vide infra). In reactions with magnesium, aluminium and gallium nitrates, the desired nitro-ester 2a was obtained in $42-59 \%$ yields (Table 1 , entries $2-4$ ). The reaction of bis(oxy)enamine 1a with lead and bismuth nitrates (Table 1, entries 5 and 6) produced formiate $\mathbf{5 a}$ in addition to dihydrooxazines $\mathbf{2 a}, \mathbf{3 a}$ and $\mathbf{4 a}$. In the case of bismuth nitrate, formiate 5 a was obtained as the major product (35\% yield). Nitrates of early transition metals afforded nitrate ester $\mathbf{2 a}$ in

Table 1 Interaction of model bis(oxy)enamine 1a with metal nitrates

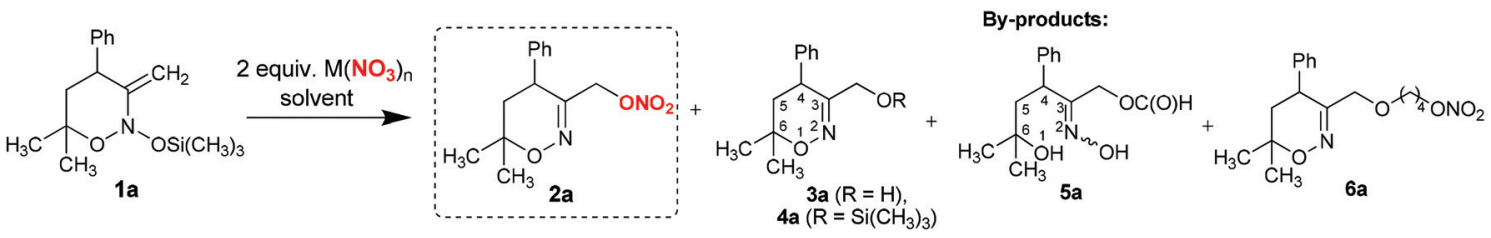

\begin{tabular}{|c|c|c|c|c|c|c|c|c|}
\hline \multirow[b]{2}{*}{ Entry } & \multirow[b]{2}{*}{ Metal nitrate $\mathrm{M}\left(\mathrm{NO}_{3}\right)_{n}$} & \multirow[b]{2}{*}{ Solvent } & \multirow[b]{2}{*}{ Time (h) } & \multirow[b]{2}{*}{ Yield of $2 \mathbf{a}^{a}(\%)$} & \multicolumn{4}{|c|}{ Yields of by-products ${ }^{a}(\%)$} \\
\hline & & & & & $3 a$ & $4 a$ & $5 \mathbf{a}$ & $6 a$ \\
\hline 1 & $\mathrm{LiNO}_{3}$ & $\mathrm{THF} / \mathrm{CH}_{2} \mathrm{Cl}_{2}$ & 2.5 & $17^{b}$ & - & 8 & - & - \\
\hline 2 & $\mathrm{Mg}\left(\mathrm{NO}_{3}\right)_{2} \cdot 6 \mathrm{H}_{2} \mathrm{O}$ & $\mathrm{THF} / \mathrm{CH}_{2} \mathrm{Cl}_{2}$ & 2.5 & 42 & 17 & 7 & - & - \\
\hline 3 & $\mathrm{Al}\left(\mathrm{NO}_{3}\right)_{3} \cdot 9 \mathrm{H}_{2} \mathrm{O}$ & $\mathrm{DMF} / \mathrm{CH}_{2} \mathrm{Cl}_{2}$ & 2.5 & 53 & 43 & - & - & - \\
\hline 4 & $\mathrm{Ga}\left(\mathrm{NO}_{3}\right)_{3} \cdot 9 \mathrm{H}_{2} \mathrm{O}$ & $\mathrm{THF} / \mathrm{CH}_{2} \mathrm{Cl}_{2}$ & 2.5 & 59 & 19 & - & - & - \\
\hline 5 & $\mathrm{~Pb}\left(\mathrm{NO}_{3}\right)_{2}$ & $\mathrm{DMF} / \mathrm{CH}_{2} \mathrm{Cl}_{2}$ & 2.5 & 40 & 23 & 18 & 4 & - \\
\hline 6 & $\mathrm{Bi}\left(\mathrm{NO}_{3}\right)_{3} \cdot 5 \mathrm{H}_{2} \mathrm{O}$ & $\mathrm{DMF} / \mathrm{CH}_{2} \mathrm{Cl}_{2}$ & 2.5 & 22 & - & - & 35 & - \\
\hline 7 & $\mathrm{Mn}\left(\mathrm{NO}_{3}\right)_{2} \cdot \mathrm{H}_{2} \mathrm{O}$ & $\mathrm{THF} / \mathrm{CH}_{2} \mathrm{Cl}_{2}$ & 2.5 & 42 & 19 & 18 & - & 4 \\
\hline 8 & $\mathrm{Fe}\left(\mathrm{NO}_{3}\right)_{3} \cdot 9 \mathrm{H}_{2} \mathrm{O}$ & $\mathrm{THF} / \mathrm{CH}_{2} \mathrm{Cl}_{2}$ & 2.5 & $43^{c}$ & - & - & - & - \\
\hline 9 & $\mathrm{Co}\left(\mathrm{NO}_{3}\right)_{2} \cdot 6 \mathrm{H}_{2} \mathrm{O}$ & $\mathrm{THF} / \mathrm{CH}_{2} \mathrm{Cl}_{2}$ & 2.5 & 59 & 32 & - & - & 6 \\
\hline 10 & $\mathrm{Co}\left(\mathrm{NO}_{3}\right)_{2}$ & $\mathrm{THF} / \mathrm{CH}_{2} \mathrm{Cl}_{2}$ & 3 & 55 & - & - & - & 6 \\
\hline 11 & $\mathrm{Ni}\left(\mathrm{NO}_{3}\right)_{2} \cdot 6 \mathrm{H}_{2} \mathrm{O}$ & $\mathrm{THF} / \mathrm{CH}_{2} \mathrm{Cl}_{2}$ & 3 & 60 & 29 & - & - & 6 \\
\hline 12 & $\mathrm{Cu}\left(\mathrm{NO}_{3}\right)_{2} \cdot 3 \mathrm{H}_{2} \mathrm{O}$ & $\mathrm{THF} / \mathrm{CH}_{2} \mathrm{Cl}_{2}$ & 2.5 & 54 & 11 & 5 & - & - \\
\hline 13 & $\mathrm{Zn}\left(\mathrm{NO}_{3}\right)_{2} \cdot 6 \mathrm{H}_{2} \mathrm{O}$ & $\mathrm{THF} / \mathrm{CH}_{2} \mathrm{Cl}_{2}$ & 3 & 53 & 32 & 7 & - & - \\
\hline 14 & $\mathrm{Cr}\left(\mathrm{NO}_{3}\right)_{3} \cdot 9 \mathrm{H}_{2} \mathrm{O}$ & $\mathrm{THF} / \mathrm{CH}_{2} \mathrm{Cl}_{2}$ & 2.5 & 71 & 21 & - & - & 6 \\
\hline 15 & $\mathrm{Cr}\left(\mathrm{NO}_{3}\right)_{3} \cdot 9 \mathrm{H}_{2} \mathrm{O}$ & $\mathrm{DMF} / \mathrm{CH}_{2} \mathrm{Cl}_{2}$ & 2.5 & 41 & 25 & 5 & - & - \\
\hline 16 & $\mathrm{AgNO}_{3}$ & $\mathrm{DMF} / \mathrm{CH}_{2} \mathrm{Cl}_{2}$ & 2.5 & 10 & 6 & - & - & - \\
\hline 17 & $\mathrm{Y}\left(\mathrm{NO}_{3}\right)_{3} \cdot 6 \mathrm{H}_{2} \mathrm{O}$ & $\mathrm{THF} / \mathrm{CH}_{2} \mathrm{Cl}_{2}$ & 3 & 24 & 56 & - & - & 7 \\
\hline 18 & $\mathrm{La}\left(\mathrm{NO}_{3}\right)_{3} \cdot 6 \mathrm{H}_{2} \mathrm{O}$ & $\mathrm{THF} / \mathrm{CH}_{2} \mathrm{Cl}_{2}$ & 2.5 & 16 & 60 & 13 & - & - \\
\hline 19 & $\mathrm{Eu}\left(\mathrm{NO}_{3}\right)_{3} \cdot 6 \mathrm{H}_{2} \mathrm{O}$ & $\mathrm{THF} / \mathrm{CH}_{2} \mathrm{Cl}_{2}$ & 2.5 & 21 & 56 & 4 & - & 6 \\
\hline 20 & $\mathrm{NH}_{4} \mathrm{NO}_{3}$ & $\mathrm{DMF} / \mathrm{CH}_{2} \mathrm{Cl}_{2}$ & 1 & 35 & 24 & 14 & - & - \\
\hline 21 & $\mathrm{HNO}_{3}$ & $\mathrm{THF} / \mathrm{CH}_{2} \mathrm{Cl}_{2}$ & 2.5 & 58 & 13 & 5 & - & - \\
\hline 22 & 3 equiv. $\mathrm{KNO}_{3}+1$ equiv. $\mathrm{BF}_{3} \cdot \mathrm{Et}_{2} \mathrm{O}$ & $\mathrm{DMF} / \mathrm{CH}_{2} \mathrm{Cl}_{2}$ & 2.5 & 26 & 37 & - & - & - \\
\hline 23 & $\mathrm{LiNO}_{3}+10 \mathrm{~mol} \% \mathrm{Cr}\left(\mathrm{NO}_{3}\right)_{3} \cdot 9 \mathrm{H}_{2} \mathrm{O}$ & $\mathrm{THF} / \mathrm{CH}_{2} \mathrm{Cl}_{2}$ & 3 & $56^{d}$ & 4 & 18 & - & 5 \\
\hline
\end{tabular}

${ }^{a}$ Yields of all products were determined by ${ }^{1} \mathrm{H}$ NMR with an internal standard. ${ }^{b}$ Conversion $-27 \%$. ${ }^{c}$ By-products were not identified.

${ }^{d}$ Conversion $-82 \%$. 
moderate to good yields, while by-product 3a was usually formed in 10-30\% yield (Table 1, entries 7-16). It should be noted that employing anhydrous nitrates allows for the suppression of the formation of $\mathbf{3 a}$, but does not lead to the increase of the yield of nitrate $2 \mathrm{a}$ due to the formation of unidentified by-products ( $c f$. entries 9 and 10 in Table 1 ).

The best result was achieved with chromium(III) nitrate nonahydrate, which gave product $2 \mathrm{a}$ in $71 \%$ yield (Table 1, entry 14). Changing of solvent to dimethylformamide led to a decrease of nitrate 2a yield (Table 1, entry 15).

Yttrium, lanthanum and europium nitrates produced 3-hydroxymethyl-substituted 1,2-oxazine $\mathbf{3 a}$ as the major product, while the yield of target 2a did not exceed $24 \%$ (Table 1, entries 17-19). The formation of nitrate ester 2a was also observed in reactions of enamine 1a with ammonium nitrate and nitric acid (yield of $35 \%$ and 58\% respectively, entries 20 and 21 in Table 1). Our attempts to use other nonmetal based Lewis acids such as boron trifluoride to promote the addition of nitrate to enamine $1 \mathrm{a}$ were not successful and led to $\mathbf{3 a}$ as the major product (entry 22 in Table 1).

Reaction with chromium nitrate was subsequently studied in more detail. UV-Vis titration of chromium nitrate solution in THF with bis(oxy)enamine 1a revealed that the majority of chromium is present in the form of the aqueous complex $\left[\mathrm{Cr}\left(\mathrm{H}_{2} \mathrm{O}\right)_{6}\right]^{3+}$ (see the ESI $\dagger$ ). This implies that a catalytic process could be designed using a catalytic amount of $\mathrm{Cr}\left(\mathrm{NO}_{3}\right)_{3}$, in combination with a cheaper nitrate source. Indeed, addition of $10 \mathrm{~mol} \%$ of chromium nitrate in the reaction of $1 \mathrm{a}$ with $\mathrm{LiNO}_{3}$ led to a high conversion of the starting material ( $c f$. entries 1 and 23 in Table 1). However, the yield of target 2 a was only $56 \%$ due to the formation of significant amounts of byproducts.

Using the procedure with chromium nitrate in THF (procedure i, Table 2), a series of 3-nitroxymethyl-substituted 1,2oxazines and isoxazolines $\mathbf{2} \mathbf{a}-\mathbf{j}$ from the corresponding bis(oxy)enamines $\mathbf{1 a}-\mathbf{j}$ were prepared. As can be seen from Table 2, the reaction is well-tolerated with various substituents in bis(oxy)enamines $\mathbf{1}$ and usually provides products in good yields. The structure of compound $2 \mathrm{~g}$ was confirmed by singlecrystal X-ray diffraction analysis.

Similar to 1a, the major by-products in reactions with bis(oxy)enamines 1b-i were 3-hydroxymethyl-substituted 1,2oxazines 3 (typically, not more than 15\%) and products of tetrahydrofuran insertion 6 (5-10\%), which in some cases were isolated and characterized. In the case of bis(oxy)enamine $\mathbf{1} \mathbf{j}$, ether $\mathbf{6 j}$ was isolated in $\mathbf{1 8 \%}$ yield. Furthermore, diether $\mathbf{7 j}$ resulting from formal insertion of two tetrahydrofuran molecules was detected in trace amounts by high-resolution massspectrometry (Table 2).

The synthesis of nitrates 2 can be accomplished directly from the corresponding cyclic nitronates $\mathbf{8}$, which are synthetic precursors of bis(oxy)enamines 1 (Scheme 2, procedure ii). In this procedure, bis(oxy)enamines are generated in situ by silylation of nitronates 8 with trimethylsilyl triflate and triethylamine, followed by treatment of the reaction mixture with chromium nitrate solution in tetrahydrofuran. Such a one-pot
Table 2 Reaction of cyclic bis(oxy)enamines $1 \mathrm{a}-\mathrm{j}$ with chromium nitrate
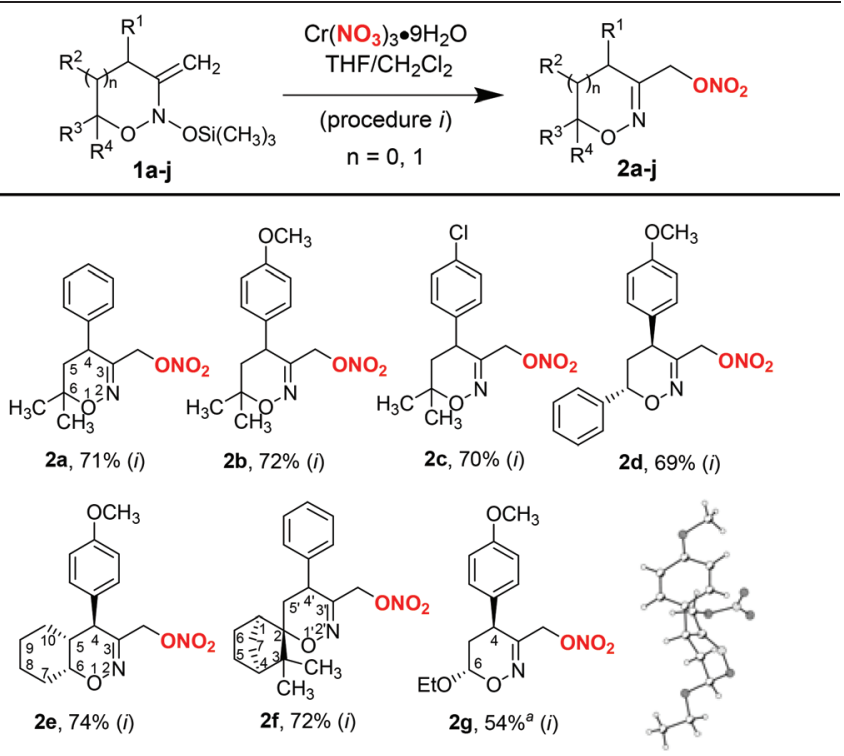

2b, $72 \%(i)$

$2 \mathrm{c}, 70 \%(i)$

2d, $69 \%(i)$
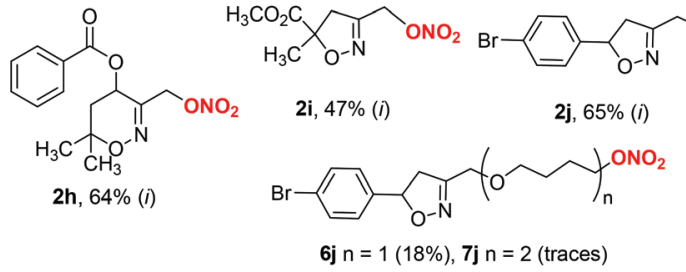

${ }^{a}$ The product contains a small amount of the 4,6-cis-isomer ( $\left.\mathrm{dr} 13: 1\right)$, probably formed via acid-promoted epimerization of acetal center C-6. ${ }^{14}$
(1)<smiles>CC1=[N+]([O-])OC(C)(C)CC1c1ccccc1</smiles>

$8 a$

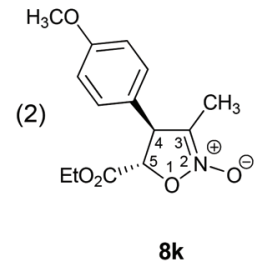

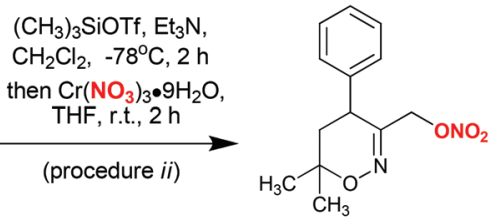

2a, $62 \%(6 a, 5 \%)$
Scheme 2 One-pot synthesis of nitrates 2 from nitronates 8 .

process is operationally simpler, however the yield of product 2a is somewhat lower compared to a two-step procedure with isolation of bis(oxy)enamine 1a ( $c f$. Scheme 2, eqn (1) and entry 14 in Table 1). Still, the application of a one-pot 
procedure is beneficial in cases when bis(oxy)enamine $\mathbf{1}$ is labile upon isolation (for example bis(oxy)enamine generated from nitronate $\mathbf{8 k}$, see Scheme 2, eqn (2)).

Procedure i did not prove to be applicable to acyclic bis(silyloxy)enamines 1l-o (Schemes 3 and 4). In the corresponding experiments, only the formation of indecipherable product mixtures was observed. Interestingly, switching to anhydrous cobalt nitrate (procedure iii) in reaction with bis(oxy)enamine 11 produced the desired labile trimethylsilyl ether of $\alpha$-nitroxyoxime 21 in 65\% yield (Scheme 3). However, in reactions with acyclic bis(silyloxy)enamines $\mathbf{1 m}, \mathbf{o}$ the corresponding nitrates $2 \mathbf{m}, \mathbf{o}$ were identified in reaction mixtures by ${ }^{1} \mathrm{H}$ NMR and GC-MS in trace amounts (see the ESI $\dagger$ ).

By conducting reactions with $\mathrm{Co}\left(\mathrm{NO}_{3}\right)_{2}$ in DMF, followed by desilylation with aqueous $\mathrm{NaHSO}_{4}$ solution, labile $\alpha$-nitroxyoximes 9 were obtained in reasonable yields (Scheme 4). In particular, by using this procedure, the simplest $\alpha$-nitroxyoxime 9m (2-(hydroxyimino)ethyl nitrate) was obtained from bis(oxy)enamine $\mathbf{1 m}$ available from nitroethane. This product can be considered as a prospective intermediate in the synthesis of high-energy compounds. $\alpha$-Nitroxy-oximes $\mathbf{9 m - 0}$ are viscous liquids, which are unstable upon heating and slowly decompose at room temperature.

The mechanism of $\alpha$-nitroxy-oxime 2 formation deserves special discussion. In fact, depending on the nature of metal nitrate, the mechanism can be different. It is likely that the process involves initial Lewis acid-mediated cleavage of weak $\mathrm{N}-\mathrm{O}$ bonds in initial bis(oxy)enamine $\mathbf{1}$, and the subsequent addition of the nitrate anion to the resulting $N$-vinyl- $N$-alkoxynitrenium cation $\mathbf{A}^{15}$ (Scheme 5 ). This is confirmed by isolation of products $\mathbf{5}$ and $\mathbf{6}$, which result from competitive addition of solvent to cation A. Thus, nitrates 2 are probably

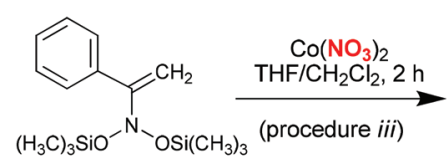

11

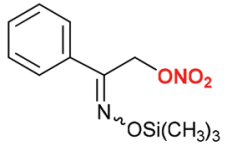

2I, $65 \%(E / Z=1: 9)$
Scheme 3 Synthesis of silyl ether $2 \mathbf{l}$ from bis(oxy)enamine $1 \mathrm{l}$.

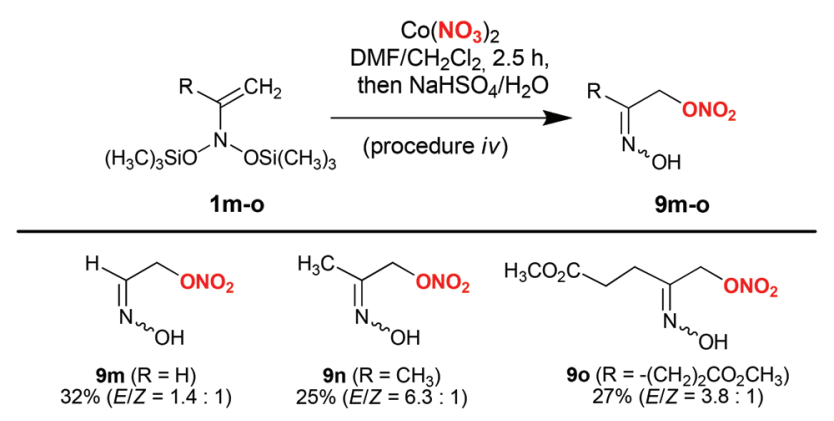

Scheme 4 Synthesis of $\alpha$-nitroxy-oximes $9 m$-o from enamines $1 \mathrm{~m}-0$.

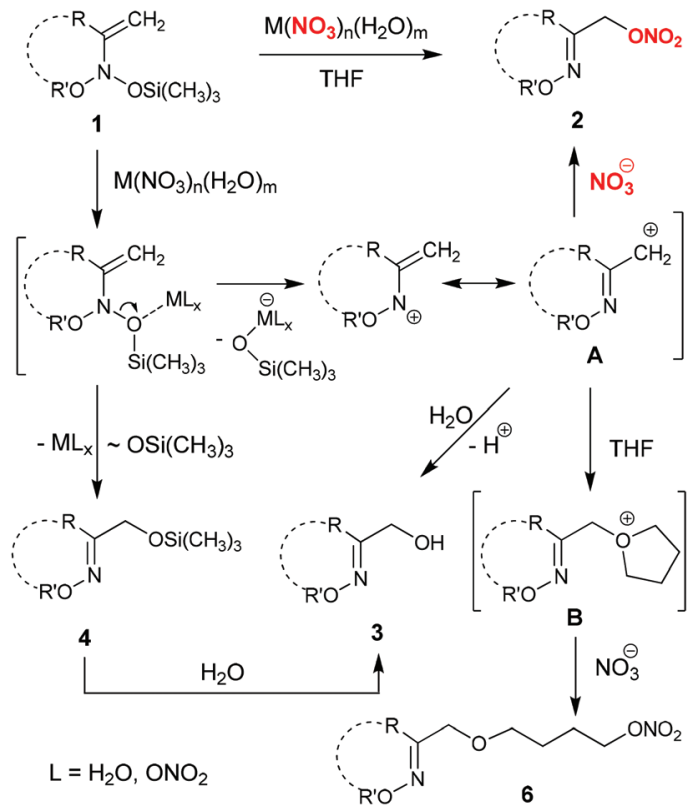

Scheme 5 Plausible mechanism for the formation of $\alpha$-nitroxy-oxime ethers 2 from bis(oxy)enamines 1.

formed as a result of $S_{N} 1^{\prime}$ substitution of the trimethylsilyloxy group in bis(oxy)enamines $\mathbf{1}$ for the nitrate anion.

The observed formation of $\alpha$-hydroxy-oxime silyl ethers 4 can be explained by LA-mediated 1,3-migration of the trimethylsilyloxy group in initial bis(oxy)enamines $1 .{ }^{15 d}$ On the other hand, $\alpha$-hydroxy-oxime cyclic ethers 3 may originate from either the addition of water to cation $\mathbf{A}$ or the hydrolysis of the corresponding trimethylsilyl ethers 4 (Scheme 5). The formation of an open-chain oxime 5a in the reaction of $\mathbf{1 a}$ with bismuth and lead nitrates (Table 1, entries 5 and 6) demonstrates the possibility of competitive coordination of these metals by endo- and exo-cyclic oxygen atoms in cyclic bis(oxy) enamines $\mathbf{1}$.

Nitrate esters are frequently employed as intermediates in the total synthesis of pharmacologically relevant compounds. . $^{3,4 b, c, 7 b, c, 8 a, b, 16}$ Recently, we developed the synthesis of oxazolidinone CMPO (a highly potent phosphodiesterase 4 inhibitor) via the nitrate intermediate $2 \mathbf{p}$ (Scheme 6). ${ }^{17 a}$ The synthesis of nitrate $\mathbf{2 p}$ was achieved by the nucleophilic substitution of bromine in 3-bromomethyl-substituted 1,2-oxazine 10p. The latter was synthesized from cyclic nitronate $\mathbf{8 p}$ with poor yield and conversion, as well as a high degree of epimerization of the acetal center C-6 (Scheme 6). ${ }^{17 a}$ Employing the process developed here, we were able to optimize the synthesis of nitrate $\mathbf{2 p}$ from nitronate $\mathbf{8} \mathbf{p}$ by the silylation of the latter and treatment of the resulting bis(oxy)enamine $\mathbf{1 p}$ with chromium nitrate. In this sequence, the yield of product $2 p$ is considerably higher and the degree of epimerization of C-6 is lower ( $c f$. both approaches in Scheme 6). Unfortunately, onepot synthesis of nitrate $2 \mathbf{p}$ from nitronate $\mathbf{8 p}$ using procedure ii was less efficient than the two-step procedure via isolation of enamine 1p (Scheme 6). 


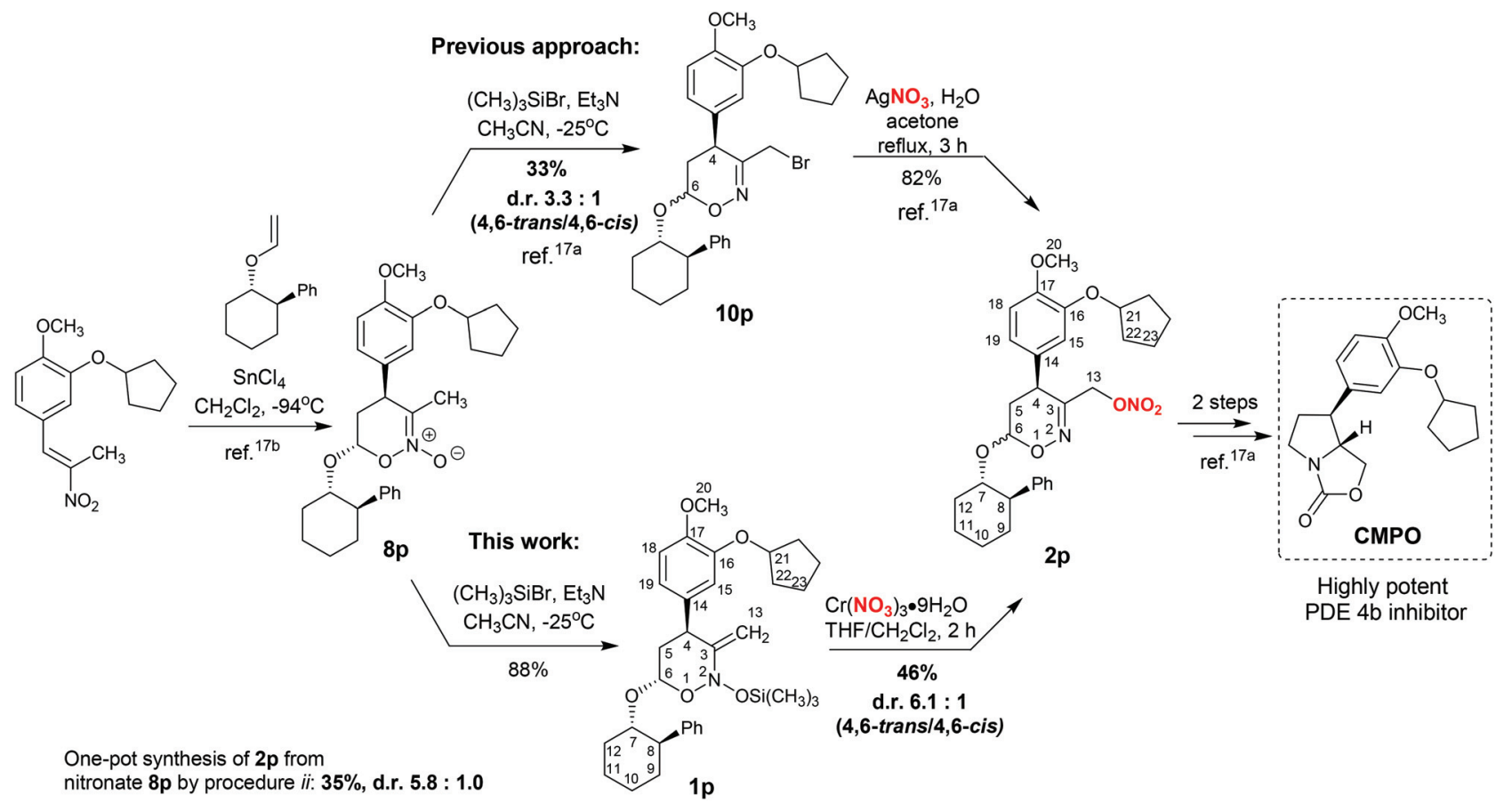

Scheme 6 Two approaches to the synthesis of nitrate $2 p-$ key precursor of the PDE $4 \mathrm{~b}$ inhibitor CMPO.

\section{Conclusions}

In conclusion, we have demonstrated that the reaction of readily available $N, N$-bis(oxy)enamines with metal nitrates results in the addition of a nitrate anion to a double carboncarbon bond, with the elimination of the trimethylsilyloxygroup from the nitrogen atom. Using reactions of bis(oxy) enamines with chromium and cobalt nitrates, a general method for the synthesis of poorly accessible $\alpha$-nitroxy-oximes of cyclic and acyclic structures was developed. These oximes are prospective NO donors and intermediates in the synthesis of bioactive molecules. The simplest $\alpha$-nitroxy-oxime, 2-(hydroxyimino)ethyl nitrate, a potential intermediate in the synthesis of high energy materials, has been synthesized in two steps from nitroethane. The efficiency of the suggested method for the total synthesis of bioactive compounds was demonstrated by the optimization of the synthesis of the highly potent PDE 4 inhibitor.

\section{Experimental}

All reactions were performed in oven-dried $\left(150^{\circ} \mathrm{C}\right)$ glassware. Tetrahydrofuran was distilled first from $\mathrm{LiAlH}_{4}$, stored under sodium benzophenone ketyl and distilled using the vacuum trap-to-trap technique prior to use. $\mathrm{CH}_{2} \mathrm{Cl}_{2}, \mathrm{MeCN} \mathrm{Et}_{3} \mathrm{~N}$, and $\left(\mathrm{CH}_{3}\right)_{3} \mathrm{SiBr}$ were distilled from $\mathrm{CaH}_{2}$. Hexane and EtOAc were distilled without drying agents. Pentane was commercial grade and used as received.

Inorganic reagents were commercial grade and were used as received. Anhydrous cobalt(II) nitrate was prepared by thermal decomposition of the corresponding hexahydrate as described in ref. 18. Initial cyclic nitronates $8 \mathbf{a}^{10 c}$ and $\mathbf{8} \mathbf{p}^{17 b}$ were synthesized according to literature methods. Bis(oxy)enamines $1 \mathbf{1 a},{ }^{10 c} \mathbf{b},{ }^{10 h} \mathbf{c},{ }^{10 g} \mathbf{d},{ }^{10 f} \mathbf{e},{ }^{10 c} \mathbf{f},{ }^{14} \mathbf{g},{ }^{10 c} \mathbf{h},{ }^{10 f} \mathbf{i},{ }^{10 i} \mathbf{j},{ }^{10 i} \mathbf{1 l},{ }^{10 e}$ and $1 \mathbf{m}-\mathbf{o}^{10 d}$ were obtained by silylation of the corresponding nitronates according to previously published procedures.

Column chromatography was performed using Kieselgel $40-60 \mu \mathrm{m} 60 \mathrm{~A}$. Analytical thin-layer chromatography was performed on silica gel plates with QF-254. Visualization was accomplished with UV light and a solution of anisaldehyde/ $\mathrm{H}_{2} \mathrm{SO}_{4}$ in ethanol.

1D and 2D NMR spectra were recorded at room temperature in $\mathrm{CDCl}_{3}$ on a Bruker AM 300 spectrometer. The chemical shifts $\left({ }^{1} \mathrm{H},{ }^{13} \mathrm{C}\right)$ are given in $\operatorname{ppm}(\delta)$ relative to the solvent signal, and liquid ammonia was used as a reference compound in the ${ }^{14} \mathrm{~N}$ NMR spectra. Multiplicities are indicated by $\mathrm{s}$ (singlet), d (doublet), $\mathrm{t}$ (triplet), q (quartet), $\mathrm{m}$ (multiplet), and br (broad). Trichloroethylene was used as an internal standard in quantitative NMR measurements.

FTIR spectra were recorded on a Bruker Alpha-T spectrometer. Peaks in IR-spectra data are reported in $\mathrm{cm}^{-1}$ with the following relative intensities: s (strong), m (medium), w (weak), br (broad), and sh (shoulder). UV-VIS spectra were recorded on a Shimadzu UVmini-1240 spectrometer (data are reported in $\mathrm{nm}$ ). Elemental analysis (average of two combustions) was performed by the Analytical Laboratory of the Institute of Organic Chemistry. HRMS were measured on an electrospray ionization (ESI) instrument with a time-of-flight (TOF) detector. GC-MS was performed on a Chromatec 5000 with the Agilent DB-1MS column 122-0132. EI mass spectra were recorded on a Finnigan MAT Incos 50 spectrometer $(70 \mathrm{eV})$. Optical rotation 
angles were measured on a JASCO P2000 polarimeter. Concentrations $c$ are given in $\mathrm{g}$ per $100 \mathrm{~mL}$. $[\alpha]_{\mathrm{D}}$ values are given in $10^{-1} \mathrm{deg} \mathrm{cm}^{2} \mathrm{~g}^{-1}$.

rel-(4S,6S)-4-(3-(Cyclopentyloxy)-4-methoxyphenyl)3-methylene-6-((1S,2R)-2-phenylcyclohexyloxy)2-(trimethylsilyloxy)morpholine (1p)

To a stirred solution of nitronate $\mathrm{rac}-\mathbf{8}^{\mathbf{1 7 b}}(0.20 \mathrm{~g}$, $0.416 \mathrm{mmol})$ and $\mathrm{Et}_{3} \mathrm{~N}(0.14 \mathrm{~mL}, 1.0 \mathrm{mmol})$ in $4.8 \mathrm{~mL}$ of $\mathrm{CH}_{2} \mathrm{Cl}_{2}$ was added $\mathrm{Me}_{3} \mathrm{SiBr}(0.12 \mathrm{~mL}, 0.91 \mathrm{mmol})$ at $-78{ }^{\circ} \mathrm{C}$ under an argon atmosphere. The mixture was kept at $-78{ }^{\circ} \mathrm{C}$ for $60 \mathrm{~h}$, then diluted with hexane $(5 \mathrm{~mL})$ and poured into a mixture of hexane $(50 \mathrm{~mL})$ and $0.25 \mathrm{M}$ aqueous $\mathrm{NaHSO}_{4}$ solution $(50 \mathrm{~mL})$. The aqueous layer was back-extracted with hexane $(20 \mathrm{~mL})$. Combined organic layers were washed with water $(30 \mathrm{~mL})$, and brine $(30 \mathrm{~mL})$, dried $\left(\mathrm{Na}_{2} \mathrm{SO}_{4}\right)$, and evaporated in a vacuum. The residue was dried in a vacuum until constant weight to give $0.202 \mathrm{~g}(88 \%)$ of bis(oxy)enamine $1 \mathrm{p}$ as colorless oil (unstable at r.t.). ${ }^{1} \mathrm{H} \mathrm{NMR}\left(\mathrm{CDCl}_{3}, 300.13 \mathrm{MHz}\right.$, COSY, HSQC): 0.31 (s, $\left.9 \mathrm{H},\left(\mathrm{CH}_{3}\right)_{3} \mathrm{Si}\right), 1.25-1.47,1.50-1.63$ and 1.72-2.00 (3 m, $16 \mathrm{H}, \mathrm{CH}-5, \mathrm{CH}_{2}-9, \mathrm{CH}_{2}-10, \mathrm{CH}_{2}-11, \mathrm{CH}-12$, $\mathrm{CH}_{2}-22, \mathrm{CH}_{2}-23$ ), 2.10 (ddd, $J=12.0,8.9,4.3 \mathrm{~Hz}, 1 \mathrm{H}, \mathrm{HC}-5$ ), 2.40 (m, $1 \mathrm{H}, H \mathrm{C}-12$ ), 2.67 (ddd, $J=11.5,10.3,2.9 \mathrm{~Hz}, 1 \mathrm{H}$, $H_{\text {ax }} \mathrm{C}-8$ ), 3.53 (br s, $\left.1 \mathrm{H}, \mathrm{HC}-4\right), 3.84$ (s, $3 \mathrm{H}, \mathrm{OCH}_{3}$ ), 3.94 (br s, $1 \mathrm{H}, \mathrm{HC}-13$ ), 4.09 (ddd, $\left.J=10.3,10.1,3.5 \mathrm{~Hz}, 1 \mathrm{H}, H_{\mathrm{ax}} \mathrm{C}-7\right), 4.75$ (br m, $2 \mathrm{H}, H \mathrm{C}-13$ and $H \mathrm{C}-21), 5.42$ (dd, $J=4.6,4.3 \mathrm{~Hz}, 1 \mathrm{H}$, $\left.H_{\mathrm{eq}} \mathrm{C}-6\right), 6.68$ (d, $\left.J=8.0 \mathrm{~Hz}, 1 \mathrm{H}, H \mathrm{C}-19\right), 6.69$ (s, $\left.1 \mathrm{H}, H \mathrm{C}-15\right)$, 6.81 (d, $J=8.0 \mathrm{~Hz}, 1 \mathrm{H}, H \mathrm{C}-18), 7.19-7.37$ (m, $5 \mathrm{H}, o^{-}, m^{-}, p^{-}$ $\left.\mathrm{C}_{6} \mathrm{H}_{5}\right) \cdot{ }^{29} \mathrm{Si} \mathrm{NMR}\left(\mathrm{CDCl}_{3}, 59.63 \mathrm{MHz}\right): 25.9 .{ }^{13} \mathrm{C} \mathrm{NMR}\left(\mathrm{CDCl}_{3}\right.$, $\left.75.47 \mathrm{MHz}, \mathrm{HSQC}):-0.51\left(\mathrm{CH}_{3}\right)_{3} \mathrm{Si}\right), 24.0,24.8$ and 26.2 $\left(\mathrm{CH}_{2}-9, \mathrm{CH}_{2}-10\right.$ and $\left.\mathrm{CH}_{2}-23\right), 30.6\left(\mathrm{CH}_{2}-12\right), 32.8\left(\mathrm{CH}_{2}-22\right), 34.5$ and $36.9\left(\mathrm{CH}_{2}-5\right.$ and $\left.\mathrm{CH}_{2}-11\right), 40.0$ (br, $\left.\mathrm{CH}-4\right), 50.2(\mathrm{CH}-8), 56.1$ $\left(\mathrm{OCH}_{3}\right), 76.2(\mathrm{CH}-7), 80.4(\mathrm{CH}-21), 93.9(\mathrm{CH}-6), 96.9$ (br, $\left.\mathrm{CH}_{2}-3\right), 111.9$ ( $\left.\mathrm{CH}-18\right), 116.0(\mathrm{CH}-15), 120.7$ ( $\left.\mathrm{CH}-19\right), 125.8$, 127.9 and $128.0\left(o-, m-, p-C_{6} \mathrm{H}_{5}\right), 132.3$ (br, $\left.C-14\right), 144.2$ $\left(\mathrm{i}-C_{6} \mathrm{H}_{5}\right), 147.4$ and $149.0(C-16$ and $C-17), 159.3$ (br, $\left.C-13\right)$. ${ }^{29} \mathrm{Si} \mathrm{NMR}\left(\mathrm{CDCl}_{3}, 59.63 \mathrm{MHz}\right): 26.0$. HRMS: $\mathrm{m} / \mathrm{z}$ Calcd for $\left[\mathrm{C}_{32} \mathrm{H}_{46} \mathrm{NO}_{5} \mathrm{Si}^{+}\right] 552.3140\left([\mathrm{M}+\mathrm{H}]^{+}\right)$. Found: 552.3136 .

rel-(4S,5S)-5-(Ethoxycarbonyl)-4-(4-methoxyphenyl)-3-methyl4,5-dihydroisoxazole 2-oxide (8k)

To a stirred solution of sulfonium ylide ${ }^{19}\left(5 \mathrm{~mL}\right.$ in $\mathrm{CHCl}_{3}, c=$ $0.14 \mathrm{~g} \mathrm{~mL} \mathrm{~m}^{-1}, 4.8 \mathrm{mmol}$ ) derived from carbethoxymethyldimethylsulfonium bromide and $\mathrm{K}_{2} \mathrm{CO}_{3}$ was added (E)-1methoxy-4-(2-nitroprop-1-enyl)benzene (0.776 g, $4 \mathrm{mmol})$. After $2.5 \mathrm{~h}$ at r.t., an additional $2.5 \mathrm{ml}$ of sulfonium ylide solution in $\mathrm{CHCl}_{3}$ was added ( $c=0.14 \mathrm{~g} \mathrm{~mL}^{-1}, 2.4 \mathrm{mmol}$ ). After $2 \mathrm{~h}$ the resulting solution was poured into a mixture of EtOAc $(100 \mathrm{~mL})$ and water $(100 \mathrm{~mL})$. The aqueous layer was backextracted with EtOAc $(50 \mathrm{~mL})$. Combined organic layers were washed with water $(50 \mathrm{~mL})$, and brine $(50 \mathrm{~mL})$, dried $\left(\mathrm{Na}_{2} \mathrm{SO}_{4}\right)$, and evaporated in a vacuum. The residue was triturated with an $\mathrm{Et}_{2} \mathrm{O}$ /pentane $(1: 10)$ mixture and dried in a vacuum until constant weight to give $0.992 \mathrm{~g}(89 \%)$ of nitronate $8 \mathbf{k}$ as a white solid. $\mathrm{Mp}=93-95{ }^{\circ} \mathrm{C} .{ }^{1} \mathrm{H} \mathrm{NMR}\left(\mathrm{CDCl}_{3}, 300.13 \mathrm{MHz}\right.$, HSQC, NOESY): 1.36 (t, $\left.J=7.1 \mathrm{~Hz}, 3 \mathrm{H}, \mathrm{CH}_{3} \mathrm{CH}_{2}\right), 1.90(\mathrm{~s}, 3 \mathrm{H}$,
$\left.\mathrm{CH}_{3}\right), 3.85\left(\mathrm{~s}, 3 \mathrm{H}, \mathrm{OCH}_{3}\right), 4.34\left(\mathrm{q}, J=7.1 \mathrm{~Hz}, 2 \mathrm{H}, \mathrm{CH}_{3} \mathrm{CH}_{2}\right)$, 4.51 (br d, $J=4.0 \mathrm{~Hz}, 1 \mathrm{H}, \mathrm{CH}-4$ ), 4.81 (d, $J=4.0 \mathrm{~Hz}, 1 \mathrm{H}$, $\mathrm{CH}-5), 6.96\left(\mathrm{~d}, J=8.6 \mathrm{~Hz}, 2 \mathrm{H}, o-\mathrm{C}_{6} \mathrm{H}_{4} \mathrm{OCH}_{3}\right), 7.21(\mathrm{~d}, J=$ $\left.8.6 \mathrm{~Hz}, 2 \mathrm{H}, m-\mathrm{C}_{6} \mathrm{H}_{4} \mathrm{OCH}_{3}\right) .{ }^{13} \mathrm{C} \mathrm{NMR}\left(\mathrm{CDCl}_{3}, 75.47 \mathrm{MHz}\right.$, HSQC): $10.6\left(\mathrm{CH}_{3}\right), 14.0\left(\mathrm{CH}_{3} \mathrm{CH}_{2}\right), 55.3\left(\mathrm{OCH}_{3}\right.$ and $\left.\mathrm{CH}-4\right), 62.2$ $\left(\mathrm{CH}_{3} \mathrm{CH}_{2}\right), 78.5(\mathrm{CH}-5), 113.3(\mathrm{C}=\mathrm{N}), 114.9\left(o-\mathrm{C}_{6} \mathrm{H}_{4} \mathrm{OCH}_{3}\right)$, $128.6\left(m-C_{6} \mathrm{H}_{4} \mathrm{OCH}_{3}\right), 128.9 \quad\left(p-C_{6} \mathrm{H}_{4} \mathrm{OCH}_{3}\right), 159.9 \quad(C-\mathrm{O})$, $168.7(C=\mathrm{O})$. Characteristic 2D NOESY correlations: $\mathrm{CH}-5 /$ $m-\mathrm{C}_{6} \mathrm{H}_{4} \mathrm{OCH}_{3}$. Anal. Calcd for $\mathrm{C}_{14} \mathrm{H}_{17} \mathrm{NO}_{5}$ : C, 60.21; H, 6.14; N, 5.02. Found: C, 60.43; H, 6.07; N, 5.05.

\section{General procedure for the synthesis of nitrates $2 a-j, p$ (procedure $i$ )}

To a stirred solution of $\mathrm{Cr}\left(\mathrm{NO}_{3}\right)_{3} \cdot 9 \mathrm{H}_{2} \mathrm{O}(0.8 \mathrm{~g}, 2.0 \mathrm{mmol})$ in THF ( $4 \mathrm{~mL}$ ) was added $2 \mathrm{~mL}$ of $0.5 \mathrm{M}$ solution of bis(oxy)enamines $\mathbf{1 a}-\mathbf{j}, \mathbf{p}$. The resulting solution was stirred for $2 \mathrm{~h}$ at room temperature and poured into a mixture of EtOAc $(50 \mathrm{~mL})$ and $0.25 \mathrm{M} \mathrm{NaHSO}_{4}$ solution $(50 \mathrm{~mL})$. The aqueous layer was backextracted with EtOAc $(50 \mathrm{~mL})$. Combined organic layers were washed with $0.25 \mathrm{M} \mathrm{NaHSO}_{4}$ solution $(50 \mathrm{~mL})$, water $(50 \mathrm{~mL})$, and brine $(50 \mathrm{~mL})$, dried $\left(\mathrm{Na}_{2} \mathrm{SO}_{4}\right)$, and evaporated in a vacuum. The residue was subjected to column chromatography on silica gel to give nitrates $\mathbf{2} \mathbf{a}-\mathbf{j}, \mathbf{p}$. For determining analytical properties, products were recrystallized from pentane $/ \mathrm{Et}_{2} \mathrm{O}$ mixtures.

General procedure for the synthesis of nitrates $2 \mathrm{a}, \mathrm{k}$ from cyclic nitronates 8 (procedure ii)

To a stirred solution of nitronates $\mathbf{8 a}, \mathbf{k}(1.0 \mathrm{mmol})$ and triethylamine $(0.17 \mathrm{~mL}, 1.2 \mathrm{mmol})$ in $\mathrm{CH}_{2} \mathrm{Cl}_{2}(3 \mathrm{~mL})$ was added trimethylsilyl triflate $(0.2 \mathrm{~mL}, 1.1 \mathrm{mmol})$ at $-78{ }^{\circ} \mathrm{C}$ under an argon atmosphere. After stirring the reaction mixture for $2 \mathrm{~h}$ at $-78{ }^{\circ} \mathrm{C}$, a solution of $\mathrm{Cr}\left(\mathrm{NO}_{3}\right)_{3} \cdot 9 \mathrm{H}_{2} \mathrm{O}(0.8 \mathrm{~g}, 2.0 \mathrm{mmol})$ in THF (4 mL) was added via a syringe and the cooling bath was removed. After $2 \mathrm{~h}$ the resulting dark-colored solution was poured into a mixture of EtOAc $(50 \mathrm{~mL})$ and $0.25 \mathrm{M} \mathrm{NaHSO}_{4}$ solution $(50 \mathrm{~mL})$. The aqueous layer was back-extracted with EtOAc $(50 \mathrm{~mL})$. Combined organic layers were washed with $0.25 \mathrm{M} \mathrm{NaHSO}_{4}$ solution $(50 \mathrm{~mL})$, water $(50 \mathrm{~mL})$, and brine $(50 \mathrm{~mL})$, dried $\left(\mathrm{Na}_{2} \mathrm{SO}_{4}\right)$, and evaporated in a vacuum. The residue was subjected to column chromatography on silica gel to give nitrates $\mathbf{2 a}, \mathbf{k}$ as well as by-products $\mathbf{6 a}$ and $\mathbf{3 k}, \mathbf{6 k}$, respectively.

(6,6-Dimethyl-4-phenyl-5,6-dihydro-4H-1,2-oxazin-3-yl)methyl nitrate (2a). Yields: 71\% (procedure i), 62\% (procedure ii). White solid. Mp 50-51 ${ }^{\circ} \mathrm{C} . R_{\mathrm{f}}=0.7$ (EtOAc/hexane $\left.=1: 1\right)$. ${ }^{1} \mathrm{H} \mathrm{NMR}\left(\mathrm{CDCl}_{3}, 300.13 \mathrm{MHz}\right): 1.31$ and $1.41\left(2 \mathrm{~s}, 6 \mathrm{H}, 2 \mathrm{CH}_{3}\right)$, $1.95\left(\mathrm{dd}, J=12.0,13.5 \mathrm{~Hz}, 1 \mathrm{H}, H_{\mathrm{ax}} \mathrm{C}-5\right), 2.13$ (dd, $J=7.8$, $\left.13.5 \mathrm{~Hz}, 1 \mathrm{H}, H_{\mathrm{eq}} \mathrm{C}-5\right), 3.51$ (dd, $J=7.8,12.0 \mathrm{~Hz}, 1 \mathrm{H}, \mathrm{H}_{\mathrm{ax}} \mathrm{C}-4$ ), 4.75 (d, $\left.J=12.4 \mathrm{~Hz}, 1 \mathrm{H}, H \mathrm{C}-\mathrm{ONO}_{2}\right), 4.84$ (d, $J=12.4 \mathrm{~Hz}, 1 \mathrm{H}$, $H \mathrm{C}-\mathrm{ONO}_{2}$ ), $7.22\left(\mathrm{~d}, J=6.7 \mathrm{~Hz}, 2 \mathrm{H}, o-\mathrm{C}_{6} H_{5}\right), 7.32-7.38(\mathrm{~m}, 3 \mathrm{H}$, $m$ - and $\left.p-\mathrm{C}_{6} \mathrm{H}_{5}\right) \cdot{ }^{13} \mathrm{C} \mathrm{NMR}\left(\mathrm{CDCl}_{3}, 75.47 \mathrm{MHz}, \mathrm{DEPT}\right): 22.6$ and $28.3\left(2 \mathrm{CH}_{3}\right), 37.8(\mathrm{CH}-4), 39.7\left(\mathrm{CH}_{2}-5\right), 72.0\left(\mathrm{CH}_{2}-\mathrm{ONO}_{2}\right), 75.7$ $(C-6), 127.9,128.1$ and $129.4\left(o-, m\right.$ - and $\left.p-C_{6} \mathrm{H}_{5}\right), 138.6$ $\left(\mathrm{i}-C_{6} \mathrm{H}_{5}\right), 151.3(C=\mathrm{N}) .{ }^{14} \mathrm{~N} \mathrm{NMR}\left(\mathrm{CDCl}_{3}, 21.69 \mathrm{MHz}\right):-45.0$. FTIR (KBr): 1643 (s, $\left.\nu_{\text {as }} \mathrm{ONO}_{2}\right)$. UV-VIS $\left(\mathrm{CH}_{2} \mathrm{Cl}_{2}\right): \lambda_{\max }, \mathrm{nm} 203$, 
237. Anal. Calcd for $\mathrm{C}_{13} \mathrm{H}_{16} \mathrm{~N}_{2} \mathrm{O}_{4}$ : C, 59.08; H, 6.10; N, 10.60 . Found: C, 59.12; H, 6.22; N, 10.53.

4-((6,6-Dimethyl-4-phenyl-5,6-dihydro-4H-1,2-oxazin-3-yl)methoxy)butyl nitrate (6a). Yield: $5 \%$ (procedure ii). Oil. Isolated as a by-product to nitrate $\mathbf{2 a}$ by column chromatography. $R_{\mathrm{f}}=0.65($ EtOAc/hexane $=1: 1) .{ }^{1} \mathrm{H} \mathrm{NMR}\left(\mathrm{CDCl}_{3}, 300.13 \mathrm{MHz}\right)$ : 1.31 and $1.35\left(2 \mathrm{~s}, 6 \mathrm{H}, 2 \mathrm{CH}_{3}\right), 1.58$ and $1.74(2 \mathrm{~m}, 4 \mathrm{H}$, $\mathrm{CH}_{2}-\mathrm{CH}_{2}$ ), 1.91 (dd, $J=12.3,13.3 \mathrm{~Hz}, 1 \mathrm{H}, H_{\mathrm{ax}} \mathrm{C}-5$ ), 2.09 (dd, $\left.J=7.9,13.3 \mathrm{~Hz}, 1 \mathrm{H}, H_{\mathrm{eq}} \mathrm{C}-5\right), 3.18$ and $3.40(2 \mathrm{~m}, 2 \mathrm{H}$, $\mathrm{CH}_{2}-\mathrm{CH}_{2}-\mathrm{O}$ ), 3.56 (dd, $J=7.9,12.3 \mathrm{~Hz}, 1 \mathrm{H}, \mathrm{CH}-4$ ), 3.81 $\left(\mathrm{s}, 2 \mathrm{H}, \mathrm{CH}_{2}-\mathrm{O}\right), 4.43\left(\mathrm{t}, J=6.5 \mathrm{~Hz}, 2 \mathrm{H}, \mathrm{CH}_{2}-\mathrm{CH}_{2}-\mathrm{ONO}_{2}\right), 7.19$ (d, $\left.J=6.7 \mathrm{~Hz}, 2 \mathrm{H}, o-\mathrm{C}_{6} H_{5}\right), 7.27-7.38\left(\mathrm{~m}, 3 \mathrm{H}, m-\right.$ and $\left.p-\mathrm{C}_{6} H_{5}\right)$. ${ }^{13} \mathrm{C} \mathrm{NMR}\left(\mathrm{CDCl}_{3}, 75.47 \mathrm{MHz}, \mathrm{DEPT}\right): 22.8$ and $28.5\left(2 \mathrm{CH}_{3}\right)$, 23.8 and $25.7\left(\mathrm{CH}_{2}-\mathrm{CH}_{2}\right), 37.4(\mathrm{CH}-4), 40.2\left(\mathrm{CH}_{2}-5\right)$, 69.6, 70.6 and $73.1\left(3 \mathrm{CH}_{2}-\mathrm{O}\right), 74.6(C-6), 127.2,128.4$ and 128.9 $\left(o-, m-\right.$ and $\left.p-C_{6} \mathrm{H}_{5}\right), 140.1\left(\mathrm{i}-C_{6} \mathrm{H}_{5}\right), 156.1(C=\mathrm{N}) .{ }^{14} \mathrm{~N}$ NMR $\left(\mathrm{CDCl}_{3}, 21.69 \mathrm{MHz}\right):$-41.1. FTIR (thin layer): 1636 $\left(\mathrm{s}, \nu_{\text {as }} \mathrm{ONO}_{2}\right.$ ). HRMS: $\mathrm{m} / z$ Calcd for $\left[\mathrm{C}_{17} \mathrm{H}_{24} \mathrm{~N}_{2} \mathrm{O}_{5} \mathrm{Na}^{+}\right] 359.1577$ $\left([\mathrm{M}+\mathrm{Na}]^{+}\right)$. Found: 359.1577 .

(4-(4-Methoxyphenyl)-6,6-dimethyl-5,6-dihydro-4H-1,2-oxazin3-yl)methyl nitrate (2b). Yield: $72 \%$ (procedure i). White solid. $\mathrm{Mp} 43-45{ }^{\circ} \mathrm{C}$ (pentane $\left./ \mathrm{Et}_{2} \mathrm{O}=5: 1\right) . R_{\mathrm{f}}=0.7$ (EtOAc $/$ hexane $=$ $1: 1) .{ }^{1} \mathrm{H} \mathrm{NMR}\left(\mathrm{CDCl}_{3}, 300.13 \mathrm{MHz}\right): 1.28$ and $1.38(2 \mathrm{~s}, 6 \mathrm{H}$, $2 \mathrm{CH}_{3}$ ), 1.91 (dd, $\left.J=12.1,13.5 \mathrm{~Hz}, 1 \mathrm{H}, H_{\mathrm{ax}} \mathrm{C}-5\right), 2.09$ (dd, $J=$ 7.8, $\left.13.5 \mathrm{~Hz}, 1 \mathrm{H}, H_{\mathrm{eq}} \mathrm{C}-5\right), 3.47$ (dd, $J=7.8,12.1 \mathrm{~Hz}, 1 \mathrm{H}$, $\left.H_{\mathrm{ax}} \mathrm{C}-4\right), 3.80\left(\mathrm{~s}, 3 \mathrm{H}, \mathrm{OCH}_{3}\right), 4.74(\mathrm{~d}, J=12.2 \mathrm{~Hz}, 1 \mathrm{H}$, $H \mathrm{C}-\mathrm{ONO}_{2}$ ), $4.80\left(\mathrm{~d}, J=12.2 \mathrm{~Hz}, 1 \mathrm{H}, H \mathrm{C}-\mathrm{ONO}_{2}\right), 6.94(\mathrm{~d}, J=$ $\left.8.7 \mathrm{~Hz}, 2 \mathrm{H}, o-\mathrm{C}_{6} \mathrm{H}_{4} \mathrm{OCH}_{3}\right), 7.11(\mathrm{~d}, J=8.7 \mathrm{~Hz}, 2 \mathrm{H}$, $\left.m-\mathrm{C}_{6} \mathrm{H}_{4} \mathrm{OCH}_{3}\right) .{ }^{13} \mathrm{C} \mathrm{NMR}\left(\mathrm{CDCl}_{3}, 75.47 \mathrm{MHz}, \mathrm{DEPT}\right): 22.6$ and $28.3\left(2 \mathrm{CH}_{3}\right), 37.0(\mathrm{CH}-4), 39.6\left(\mathrm{CH}_{2}-5\right), 55.3\left(\mathrm{OCH}_{3}\right)$, $71.9\left(\mathrm{CH}_{2}-\mathrm{ONO}_{2}\right), 75.8(C-6), 114.7 \quad\left(o-C_{6} \mathrm{H}_{4} \mathrm{OCH}_{3}\right), 129.2$ $\left(m-C_{6} \mathrm{H}_{4} \mathrm{OCH}_{3}\right), \quad 130.3 \quad\left(p-C_{6} \mathrm{H}_{4} \mathrm{OCH}_{3}\right), 151.6 \quad(C-\mathrm{O}), \quad 159.2$ $(C=\mathrm{N}) .{ }^{14} \mathrm{~N}$ NMR $\left(\mathrm{CDCl}_{3}, 21.69 \mathrm{MHz}\right):-45.0$. FTIR $(\mathrm{KBr}): 1644$ $\left(\mathrm{s}, \nu_{\text {as }} \mathrm{ONO}_{2}\right)$. Anal. Calcd for $\mathrm{C}_{14} \mathrm{H}_{18} \mathrm{~N}_{2} \mathrm{O}_{5}$ : C, 57.13; H, 6.16; N, 9.52. Found: C, 57.02; H, 6.05; N, 9.44.

(4-(4-Chlorophenyl)-6,6-dimethyl-5,6-dihydro-4H-1,2-oxazin3-yl)methyl nitrate (2c). Yield: 70\% (procedure i). White solid. Mp 59-61 ${ }^{\circ} \mathrm{C}\left(\right.$ pentane $\left./ \mathrm{Et}_{2} \mathrm{O}=5: 1\right) . R_{\mathrm{f}}=0.7$ (EtOAc/hexane $=$ $1: 1) .{ }^{1} \mathrm{H} \mathrm{NMR}\left(\mathrm{CDCl}_{3}, 300.13 \mathrm{MHz}\right): 1.29$ and $1.39(2 \mathrm{~s}, 6 \mathrm{H}$, $\left.2 \mathrm{CH}_{3}\right), 1.89$ (dd, $\left.J=12.0,13.3 \mathrm{~Hz}, 1 \mathrm{H}, H_{\mathrm{ax}} \mathrm{C}-5\right), 2.14(\mathrm{dd}, J=$ 7.8, $\left.13.3 \mathrm{~Hz}, 1 \mathrm{H}, H_{\mathrm{eq}} \mathrm{C}-5\right), 3.52(\mathrm{dd}, J=7.8,12.0 \mathrm{~Hz}, 1 \mathrm{H}$, $\left.H_{\text {ax }} \mathrm{C}-4\right), 4.72\left(\mathrm{~d}, J=12.5 \mathrm{~Hz}, 1 \mathrm{H}, H \mathrm{C}-\mathrm{ONO}_{2}\right), 4.84(\mathrm{~d}, J=$ $12.5 \mathrm{~Hz}, 1 \mathrm{H}, \mathrm{HC}-\mathrm{ONO}_{2}$ ), 7.16 (d, $\left.J=8.3 \mathrm{~Hz}, 2 \mathrm{H}, \mathrm{C}_{6} \mathrm{H}_{4} \mathrm{Cl}\right), 7.35$ (d, $\left.J=8.3 \mathrm{~Hz}, 2 \mathrm{H}, \mathrm{C}_{6} \mathrm{H}_{4} \mathrm{Cl}\right) .{ }^{13} \mathrm{C} \mathrm{NMR}\left(\mathrm{CDCl}_{3}, 75.47 \mathrm{MHz}\right.$, DEPT): 22.6 and $28.2\left(2 \mathrm{CH}_{3}\right), 37.2(\mathrm{CH}-4), 39.6\left(\mathrm{CH}_{2}-5\right), 71.8$ $\left(\mathrm{CH}_{2}-7\right), 75.7(C-6), 129.5$ and $129.6\left(o-\right.$ and $\left.m-\mathrm{C}_{6} \mathrm{H}_{4} \mathrm{Cl}\right), 133.8$ and $137.1\left(p-C_{6} \mathrm{H}_{4} \mathrm{Cl}\right.$ and $\left.C-\mathrm{Cl}\right), 150.7(C=\mathrm{N}) .{ }^{14} \mathrm{~N}$ NMR $\left(\mathrm{CDCl}_{3}, 21.69 \mathrm{MHz}\right):-45.6$. FTIR (thin layer): $1629\left(\mathrm{~s}, \nu_{\text {as }}\right.$ $\mathrm{ONO}_{2}$ ). Anal. Calcd for $\mathrm{C}_{13} \mathrm{H}_{15} \mathrm{ClN}_{2} \mathrm{O}_{4}: \mathrm{C}, 52.27 ; \mathrm{H}, 5.06 ; \mathrm{N}$, 9.38. Found: C, 52.35; H, 5.06; N, 9.30.

4-((4-(4-Chlorophenyl)-6,6-dimethyl-5,6-dihydro-4H-1,2-oxazin3-yl)methoxy)butyl nitrate (6c). Yield: $8 \%$ (procedure i). Oil. Isolated as a by-product to nitrate $2 \mathrm{c}$ by column chromatography. $R_{\mathrm{f}}=0.6($ EtOAc/hexane $=1: 1) .{ }^{1} \mathrm{H}$ NMR $\left(\mathrm{CDCl}_{3}\right.$, $300.13 \mathrm{MHz}): 1.29$ and $1.37\left(2 \mathrm{~s}, 6 \mathrm{H}, 2 \mathrm{CH}_{3}\right), 1.59$ and 1.75 ( $2 \mathrm{~m}, 4 \mathrm{H}, \mathrm{CH}_{2}-\mathrm{CH}_{2}$ ), 1.86 (dd, $J=12.1,13.3 \mathrm{~Hz}, 1 \mathrm{H}, H_{\mathrm{ax}} \mathrm{C}-5$ ), $2.08\left(\mathrm{dd}, J=7.8,13.3 \mathrm{~Hz}, 1 \mathrm{H}, H_{\mathrm{eq}} \mathrm{C}-5\right), 3.20$ and $3.38(2 \mathrm{~m}, 2 \mathrm{H}$, $\mathrm{CH}_{2}-\mathrm{CH}_{2}-\mathrm{O}$ ), 3.55 (dd, $J=7.8,12.1 \mathrm{~Hz}, 1 \mathrm{H}, \mathrm{CH}-4$ ), 3.80 (s, $\left.2 \mathrm{H}, \mathrm{CH}_{2}-\mathrm{O}\right), 4.44\left(\mathrm{t}, J=6.4 \mathrm{~Hz}, 2 \mathrm{H}, \mathrm{CH}_{2}-\mathrm{CH}_{2}-\mathrm{ONO}_{2}\right.$ ), 7.14 (d, $J=8.3 \mathrm{~Hz}, 2 \mathrm{H}, \mathrm{C}_{6} \mathrm{H}_{4} \mathrm{Cl}$ ), 7.32 (d, $J=8.3 \mathrm{~Hz}, 2 \mathrm{H}, \mathrm{C}_{6} \mathrm{H}_{4} \mathrm{Cl}$ ). ${ }^{13} \mathrm{C} \mathrm{NMR}\left(\mathrm{CDCl}_{3}, 75.47 \mathrm{MHz}, \mathrm{DEPT}\right): 22.7$ and $28.4\left(2 \mathrm{CH}_{3}\right)$, 23.8 and $25.7\left(\mathrm{CH}_{2}-\mathrm{CH}_{2}\right), 36.8(\mathrm{CH}-4), 40.0\left(\mathrm{CH}_{2}-5\right)$, 69.6, 70.5 and 73.0 ( $3 \mathrm{CH}_{2}-\mathrm{O}$ ), $74.6(C-6), 129.1$ and 129.7 (o- and $\left.m-\mathrm{C}_{6} \mathrm{H}_{4} \mathrm{Cl}\right), 133.1$ and $138.6\left(p-C_{6} \mathrm{H}_{4} \mathrm{Cl}\right.$ and $\left.C-\mathrm{Cl}\right), 155.5$ $(C=\mathrm{N}) .{ }^{14} \mathrm{~N}$ NMR $\left(\mathrm{CDCl}_{3}, 21.69 \mathrm{MHz}\right):-40.9$. FTIR $(\mathrm{KBr}): 1641$ $\left(\mathrm{s}, \quad \nu_{\mathrm{as}} \mathrm{ONO}_{2}\right)$. HRMS: $m / z$ Calcd for $\left[\mathrm{C}_{17} \mathrm{H}_{23} \mathrm{ClN}_{2} \mathrm{O}_{5} \mathrm{Na}^{+}\right]$ 393.1188 $\left([\mathrm{M}+\mathrm{Na}]^{+}\right)$. Found: 393.1182 .

(rel-(4S,6S)-4-(4-Methoxyphenyl)-6-phenyl-5,6-dihydro-4H1,2-oxazin-3-yl)methyl nitrate (2d). Yield: 69\% (procedure i). White solid. $\mathrm{Mp} 73-75{ }^{\circ} \mathrm{C}$ (pentane $/ \mathrm{Et}_{2} \mathrm{O}=5: 1$ ). $R_{\mathrm{f}}=$ 0.7 (EtOAc/hexane = 1:1). ${ }^{1} \mathrm{H}$ NMR $\left(\mathrm{CDCl}_{3}, 300.13 \mathrm{MHz}\right): 2.13$ (ddd, $J=13.6,2.1,1.5 \mathrm{~Hz}, 1 \mathrm{H}, H_{\mathrm{eq}} \mathrm{C}-5$ ), 2.38 (ddd, $J=13.6$, $\left.11.3,6.3 \mathrm{~Hz}, 1 \mathrm{H}, H_{\mathrm{ax}} \mathrm{C}-5\right), 3.66$ (dd, $J=6.3,1.5 \mathrm{~Hz}, 1 \mathrm{H}$, $\left.H_{\text {eq }} \mathrm{C}-4\right), 3.84$ (s, $3 \mathrm{H}, \mathrm{OCH}_{3}$ ), 4.91 (dd, $J=11.1,2.1 \mathrm{~Hz}, 1 \mathrm{H}$, $\left.H_{\mathrm{ax}} \mathrm{C}-6\right), 4.96$ (d, $\left.J=12.8 \mathrm{~Hz}, 1 \mathrm{H}, H \mathrm{C}-\mathrm{ONO}_{2}\right), 5.09$ (d, $J=$ $\left.12.8 \mathrm{~Hz}, 1 \mathrm{H}, \mathrm{HC}-\mathrm{ONO}_{2}\right), 6.96\left(\mathrm{~d}, J=8.3 \mathrm{~Hz}, 2 \mathrm{H}, o-\mathrm{C}_{6} \mathrm{H}_{4} \mathrm{OCH}_{3}\right)$, $7.17\left(\mathrm{~d}, J=8.3 \mathrm{~Hz}, 2 \mathrm{H}, m-\mathrm{C}_{6} \mathrm{H}_{4} \mathrm{OCH}_{3}\right), 7.31-7.42(\mathrm{~m}, 5 \mathrm{H}$, $\left.o-, m-, p-\mathrm{C}_{6} \mathrm{H}_{5}\right) .{ }^{13} \mathrm{C} \mathrm{NMR}\left(\mathrm{CDCl}_{3}, 75.47 \mathrm{MHz}, \mathrm{DEPT}\right): 34.2\left(\mathrm{CH}_{2}-5\right)$, 36.7 ( $\mathrm{CH}-4), 55.4\left(\mathrm{OCH}_{3}\right), 71.8\left(\mathrm{CH}_{2}-\mathrm{ONO}_{2}\right), 74.0(\mathrm{CH}-6), 114.8$ $\left(o-C_{6} \mathrm{H}_{4} \mathrm{OCH}_{3}\right), 126.6,128.5$ and $128.6\left(o-, m-, p-C_{6} \mathrm{H}_{5}\right), 129.3$ $\left(m-C_{6} \mathrm{H}_{4} \mathrm{OCH}_{3}\right), 132.0 \quad\left(p-C_{6} \mathrm{H}_{4} \mathrm{OCH}_{3}\right), 138.7\left(\mathrm{i}-C_{6} \mathrm{H}_{5}\right), 150.6$ $(C-\mathrm{O}), 159.2(C=\mathrm{N}) .{ }^{14} \mathrm{~N}$ NMR $\left(\mathrm{CDCl}_{3}, 21.69 \mathrm{MHz}\right):-45.8$. FTIR: $1643\left(\mathrm{~s}, \nu_{\text {as }} \mathrm{ONO}_{2}\right)$. Anal. Calcd for $\mathrm{C}_{18} \mathrm{H}_{18} \mathrm{~N}_{2} \mathrm{O}_{5}$ : C, 63.15; H, 5.30; N, 8.18. Found: C, 62.63; H, 5.26; N, 7.97.

rel-[(4S,4aR,8aR)-4-(4-Methoxyphenyl)-4a,5,6,7,8,8a-hexahydro4H-1,2-benzoxazin-3-yl]methyl nitrate (2e). Yield: 74\% (procedure i). White solid. $\mathrm{Mp} 78-80{ }^{\circ} \mathrm{C}$ (pentane $\left./ \mathrm{Et}_{2} \mathrm{O}=5: 1\right) \cdot R_{\mathrm{f}}=$ $0.8($ EtOAc/hexane $=1: 1) .{ }^{1} \mathrm{H}$ NMR $\left(\mathrm{CDCl}_{3}, 300.13 \mathrm{MHz}, \mathrm{COSY}\right.$, HSQC): $1.22-1.53,1.54-1.69$ and 1.70-1.82 (3 m, $4 \mathrm{H}, 2 \mathrm{H}$ and $\left.2 \mathrm{H}, \mathrm{HC}-5, \mathrm{HC}-7, \mathrm{H}_{2} \mathrm{C}-8, \mathrm{H}_{2} \mathrm{C}-9, \mathrm{H}_{2} \mathrm{C}-10\right)$, 2.03-2.12 (m, $1 \mathrm{H}$, HC-7), 3.12 (br s, $1 \mathrm{H}, H_{\mathrm{eq}} \mathrm{C}-4$ ), 3.80 (s, $3 \mathrm{H}, H_{3} \mathrm{CO}$ ), 4.06 (br m, $1 \mathrm{H}, H \mathrm{C}-6), 4.86\left(\mathrm{~d}, J=12.7 \mathrm{~Hz}, 1 \mathrm{H}, H \mathrm{C}-\mathrm{ONO}_{2}\right), 5.02(\mathrm{~d}, J=$ 12.7 Hz, $1 \mathrm{H}, \mathrm{HC}-\mathrm{ONO}_{2}$ ), 6.89 (d, $J=8.6 \mathrm{~Hz}, 2 \mathrm{H}, o-\mathrm{C}_{6} \mathrm{H}_{4} \mathrm{OCH}_{3}$ ), $7.07\left(\mathrm{~d}, J=8.6 \mathrm{~Hz}, 2 \mathrm{H}, m-\mathrm{C}_{6} \mathrm{H}_{4} \mathrm{OCH}_{3}\right) .{ }^{13} \mathrm{C} \mathrm{NMR}\left(\mathrm{CDCl}_{3}\right.$, $75.47 \mathrm{MHz}$, COSY, HSQC, DEPT): 19.9 and $24.8\left(\mathrm{CH}_{2}-9\right.$ and $\left.\mathrm{CH}_{2}-10\right), 27.5\left(\mathrm{CH}_{2}-8\right), 29.0\left(\mathrm{CH}_{2}-7\right), 38.8(\mathrm{CH}-5), 43.5$ $(\mathrm{CH}-4), 55.3\left(\mathrm{OCH}_{3}\right), 69.7(\mathrm{CH}-6), 72.1\left(\mathrm{CH}_{2}-\mathrm{ONO}_{2}\right), 114.5$ $\left(o-C_{6} \mathrm{H}_{4} \mathrm{OCH}_{3}\right), 129.1\left(m-C_{6} \mathrm{H}_{4} \mathrm{OCH}_{3}\right), 132.1 \quad\left(p-C_{6} \mathrm{H}_{4} \mathrm{OCH}_{3}\right)$, 148.9 (C-O), $159.0(C-3) .{ }^{14} \mathrm{~N}$ NMR $\left(\mathrm{CDCl}_{3}, 21.69 \mathrm{MHz}\right):-45.1$. FTIR (KBr): $1639\left(\mathrm{~s}, \nu_{\text {as }} \mathrm{ONO}_{2}\right)$. Anal. Calcd for $\mathrm{C}_{16} \mathrm{H}_{20} \mathrm{NO}_{5}$ : C, 59.99; H, 6.29; N, 8.74. Found: C, 60.03; H, 6.36; N, 8.73.

$\left(\left(1 R, 2 S, 4 S, 4^{\prime} S\right)-3,3\right.$-Dimethyl-4'-phenyl-4', 5'-dihydrospiro[bicyclo[2.2.1] heptane-2,6'-[1,2] oxazine]-3'-yl)methyl nitrate (2f). Yield: $72 \%$ (procedure i). White solid. Mp $65-72{ }^{\circ} \mathrm{C}$ (pentane). $R_{\mathrm{f}}=0.8($ EtOAc/hexane $=1: 1) \cdot[\alpha]_{\mathrm{D}}=+48.1$ (EtOAc, $\left.c=1.0,24{ }^{\circ} \mathrm{C}\right) .{ }^{1} \mathrm{H}$ NMR $\left(\mathrm{CDCl}_{3}, 300.13 \mathrm{MHz}, \mathrm{COSY}\right.$, HSQC): 0.91 (s, $\left.3 \mathrm{H}, \mathrm{CH}_{3}\right), 1.16$ (s, $\left.3 \mathrm{H}, \mathrm{CH}_{3}\right), 1.19$ (d, $J=10.1 \mathrm{~Hz}, 1 \mathrm{H}$, HC-7), 1.24-1.46 (m, $\left.2 \mathrm{H}, \mathrm{CH}_{2}-6\right)$, 1.48-1.64 (m, $2 \mathrm{H}, \mathrm{CH}_{2}-5$ ), 1.83 (dd, $J=13.6,12.7 \mathrm{~Hz}, 1 \mathrm{H}, H_{\text {ax }}^{\prime} \mathrm{C}-5^{\prime}$ ), 1.85 (br s, $1 \mathrm{H}, H \mathrm{C}-4$ ), 2.18 (d, $J=10.1 \mathrm{~Hz}, 1 \mathrm{H}, H \mathrm{C}-7), 2.33$ (dd, $J=13.6,7.0 \mathrm{~Hz}, 1 \mathrm{H}$, $\left.H^{\prime \prime}{ }_{\text {eq }} \mathrm{C}-5^{\prime}\right), 2.38$ (d, $\left.J=4.4 \mathrm{~Hz}, 1 \mathrm{H}, \mathrm{HC}-1\right), 3.42(\mathrm{dd}, J=12.7$, $\left.7.0 \mathrm{~Hz}, 1 \mathrm{H}, H_{\mathrm{ax}} \mathrm{C}-4^{\prime}\right), 4.74$ (d, $J=12.4 \mathrm{~Hz}, 1 \mathrm{H}, H \mathrm{C}-\mathrm{ONO}_{2}$ ), 
$4.80\left(\mathrm{~d}, J=12.4 \mathrm{~Hz}, 1 \mathrm{H}, H \mathrm{C}-\mathrm{ONO}_{2}\right), 7.22(\mathrm{~d}, J=7.7 \mathrm{~Hz}, 2 \mathrm{H}$, $\left.o-\mathrm{C}_{6} \mathrm{H}_{5}\right), 7.30-7.42\left(\mathrm{~m}, 3 \mathrm{H}, m-\right.$ and $\left.p-\mathrm{C}_{6} H_{5}\right) .{ }^{13} \mathrm{C} \mathrm{NMR}\left(\mathrm{CDCl}_{3}\right.$, 75.47 MHz, HSQC, DEPT): 22.3 and $24.2\left(2 \mathrm{CH}_{3}\right), 22.3$ and 23.8 $\left(\mathrm{CH}_{2}-5\right.$ and $\left.\mathrm{CH}_{2}-6\right), 30.8\left(\mathrm{CH}_{2}-5^{\prime}\right), 34.6\left(\mathrm{CH}_{2}-7\right), 39.9\left(\mathrm{CH}-4^{\prime}\right)$, 42.7 (CH-1), 44.7 (C-3), 49.3 (CH-4), $72.0\left(\mathrm{CH}_{2}-8\right), 87.8(C-2)$, 127.9, 128.1 and $129.4\left(o-, m-, p-C_{6} \mathrm{H}_{5}\right), 138.8\left(\mathrm{i}-C_{6} \mathrm{H}_{5}\right), 151.9$ $\left(C-3{ }^{\prime}\right) .{ }^{14} \mathrm{~N}$ NMR $\left(\mathrm{CDCl}_{3}, 21.69 \mathrm{MHz}\right):-45.0$. FTIR (KBr): 1647 (s, $\nu_{\text {as }} \mathrm{ONO}_{2}$ ). Anal. Calcd for $\mathrm{C}_{19} \mathrm{H}_{24} \mathrm{~N}_{2} \mathrm{O}_{4}$ : C, 66.26; H, 7.02; N, 8.13. Found: C, 66.28; H, 7.00; N, 8.03.

[rel-(4S,6S)-6-ethoxy-4-(4-methoxyphenyl)-5,6-dihydro-4H-1,2oxazin-3-yl]methyl nitrate (2g). Yield: $54 \%$ (procedure i). White solid. Mp 61-62 ${ }^{\circ} \mathrm{C}$ (pentane/ $\left.\mathrm{Et}_{2} \mathrm{O}=5: 1\right) . \quad R_{\mathrm{f}}=$ $0.7($ EtOAc/hexane $=1: 1) .{ }^{1} \mathrm{H}$ NMR $\left(\mathrm{CDCl}_{3}, 300.13 \mathrm{MHz}\right): 1.25$ (t, $J=7.1 \mathrm{~Hz}, 3 \mathrm{H}, \mathrm{CH}_{3}$ ), 2.12 (ddd, $J=2.6,12.0,13.5 \mathrm{~Hz}, 1 \mathrm{H}$, $H_{\text {ax }} \mathrm{C}-5$ ), 2.28 (ddd, $J=2.4,7.6,13.5 \mathrm{~Hz}, 1 \mathrm{H}, H_{\mathrm{eq}} \mathrm{C}-5$ ), 3.68 (dd, $\left.J=7.6,12.0 \mathrm{~Hz}, 1 \mathrm{H}, H_{\mathrm{ax}} \mathrm{C}-4\right), 3.63$ and $3.90(2 \mathrm{~m}, 2 \mathrm{H}$, $\left.\mathrm{OCH}_{2} \mathrm{CH}_{3}\right), 3.82\left(\mathrm{~s}, 3 \mathrm{H}, \mathrm{OCH}_{3}\right), 4.73(\mathrm{~d}, J=13.2 \mathrm{~Hz}, 1 \mathrm{H}$, $H \mathrm{C}-\mathrm{ONO}_{2}$ ), 4.81 (d, $\left.J=13.2 \mathrm{~Hz}, 1 \mathrm{H}, H \mathrm{C}-\mathrm{ONO}_{2}\right), 5.20(\mathrm{dd}, J=$ 2.4 and $\left.2.6 \mathrm{~Hz}, 1 \mathrm{H}, H_{\mathrm{eq}} \mathrm{C}-6\right), 6.91$ (d, $J=8.6 \mathrm{~Hz}, 2 \mathrm{H}$, $\left.o-\mathrm{C}_{6} \mathrm{H}_{4} \mathrm{OCH}_{3}\right), 7.13\left(\mathrm{~d}, J=8.6 \mathrm{~Hz}, 2 \mathrm{H}, m-\mathrm{C}_{6} \mathrm{H}_{4} \mathrm{OCH}_{3}\right) .{ }^{13} \mathrm{C} \mathrm{NMR}$ $\left(\mathrm{CDCl}_{3}, 75.47 \mathrm{MHz}, \mathrm{DEPT}\right): 15.0\left(\mathrm{CH}_{3} \mathrm{CH}_{2}-\mathrm{O}\right), 32.4\left(\mathrm{CH}_{2}-5\right)$, $34.0(\mathrm{CH}-4), 55.3\left(\mathrm{OCH}_{3}\right), 64.0\left(\mathrm{CH}_{3} \mathrm{CH}_{2}-\mathrm{O}\right), 71.4\left(\mathrm{CH}_{2}-\mathrm{ONO}_{2}\right)$, $96.1(C \mathrm{H}-6), 114.8\left(o-C_{6} \mathrm{H}_{4} \mathrm{OCH}_{3}\right), 129.3\left(m-C_{6} \mathrm{H}_{4} \mathrm{OCH}_{3}\right), 130.0$ $\left(p-C_{6} \mathrm{H}_{4} \mathrm{OCH}_{3}\right), 154.3(C-\mathrm{O}), 159.2(C=\mathrm{N}) .{ }^{14} \mathrm{~N} \mathrm{NMR}\left(\mathrm{CDCl}_{3}\right.$, 21.69 MHz): -45.8. FTIR ( $\mathrm{KBr}): 1643$ (s, $\left.\nu_{\text {as }} \mathrm{ONO}_{2}\right)$. Anal. Calcd for $\mathrm{C}_{14} \mathrm{H}_{18} \mathrm{~N}_{2} \mathrm{O}_{6}$ : C, 54.19; H, 5.85; N, 9.03. Found: C, 54.22; $\mathrm{H}, 5.89$; N, 8.98. Single crystals for X-ray analysis (CCDC 1419082) were obtained by crystallization from pentane/ $\mathrm{Et}_{2} \mathrm{O}=5: 1$ at $-30{ }^{\circ} \mathrm{C}$. Minor 4,6-cis-isomer: ${ }^{1} \mathrm{H} \mathrm{NMR}\left(\mathrm{CDCl}_{3}, 300.13 \mathrm{MHz}\right.$, characteristic signals): 2.45 (ddd, $J=3.1,7.8,13.8 \mathrm{~Hz}$, $\left.1 \mathrm{H}, H_{\mathrm{ax}} \mathrm{C}-5\right), 4.80\left(\mathrm{~d}, J=12.6 \mathrm{~Hz}, 1 \mathrm{H}, H \mathrm{C}-\mathrm{ONO}_{2}\right), 4.90$ (d, $J=12.6 \mathrm{~Hz}, 1 \mathrm{H}, H \mathrm{C}-\mathrm{ONO}_{2}$ ), $5.10(\mathrm{dd}, J=2.9,5.7 \mathrm{~Hz}, 1 \mathrm{H}$, $\left.H_{\text {eq }} \mathrm{C}-6\right)$.

6,6-Dimethyl-3-(nitrooxymethyl)-5,6-dihydro-4H-1,2-oxazin-4yl benzoate ( $2 \mathrm{~h})$. Yield: $64 \%$ (procedure i). Oil. $R_{\mathrm{f}}=0.7$ (EtOAc/ hexane $=1: 1) .{ }^{1} \mathrm{H} \mathrm{NMR}\left(\mathrm{CDCl}_{3}, 300.13 \mathrm{MHz}\right): 1.43$ and 1.44 $\left(2 \mathrm{~s}, 6 \mathrm{H}, 2 \mathrm{CH}_{3}\right), 2.08$ (dd, $\left.J=5.8,14.3 \mathrm{~Hz}, 1 \mathrm{H}, H \mathrm{C}-5\right), 2.33$ (dd, $J=6.6,14.3 \mathrm{~Hz}, 1 \mathrm{H}, H \mathrm{C}-5), 5.16$ (d, $J=12.7 \mathrm{~Hz}, 1 \mathrm{H}$, $\left.H \mathrm{C}-\mathrm{ONO}_{2}\right), 5.30\left(\mathrm{~d}, J=12.7 \mathrm{~Hz}, 1 \mathrm{H}, H \mathrm{C}-\mathrm{ONO}_{2}\right), 5.62(\mathrm{dd}, J=$ $5.8,6.6 \mathrm{~Hz}, 1 \mathrm{H}, H \mathrm{C}-4), 7.51$ (dd, $J=7.5,7.6 \mathrm{~Hz}, 2 \mathrm{H}, m-\mathrm{C}_{6} H_{5}$ ), $7.66\left(\mathrm{t}, J=7.5 \mathrm{~Hz}, 1 \mathrm{H}, p-\mathrm{C}_{6} H_{5}\right), 8.06(\mathrm{~d}, J=7.6 \mathrm{~Hz}, 2 \mathrm{H}$, $\left.m-\mathrm{C}_{6} \mathrm{H}_{5}\right) .{ }^{13} \mathrm{C} \mathrm{NMR}\left(\mathrm{CDCl}_{3}, 75.47 \mathrm{MHz}, \mathrm{DEPT}\right): 25.2$ and 25.9 (2 $\left.\mathrm{CH}_{3}\right), 35.9\left(\mathrm{CH}_{2}-5\right), 60.1(\mathrm{CH}-4)$, $70.7\left(\mathrm{CH}_{2}-\mathrm{ONO}_{2}\right), 75.8(C-6)$, $128.7,129.7$ and $133.8\left(o-, m-, p-C_{6} \mathrm{H}_{5}\right.$ and $\left.\mathrm{i}-C_{6} \mathrm{H}_{5}\right), 147.6$ $(C=\mathrm{N}), 165.3(C=\mathrm{O}) .{ }^{14} \mathrm{~N} \mathrm{NMR}\left(\mathrm{CDCl}_{3}, 21.69 \mathrm{MHz}\right):-46.6$. FTIR (thin layer): $1724(\mathrm{~s}, \mathrm{C}=\mathrm{O}), 1643$ (s, $\left.\nu_{\text {as }} \mathrm{ONO}_{2}\right)$. Anal. Calcd for $\mathrm{C}_{14} \mathrm{H}_{16} \mathrm{~N}_{2} \mathrm{O}_{6}$ : C, 54.54; H, 5.23; N, 9.09. Found: C, 54.40; H, 4.88; N, 8.90.

Methyl 5-methyl-3-(nitrooxymethyl)-4,5-dihydroisoxazole-5carboxylate (2i). Yield: $47 \%$ (procedure i). Oil. $R_{\mathrm{f}}=0.4$ (EtOAc/ hexane $=1: 3) .{ }^{1} \mathrm{H} \mathrm{NMR}\left(\mathrm{CDCl}_{3}, 300.13 \mathrm{MHz}\right): 1.66(\mathrm{~s}, 3 \mathrm{H}$, $\mathrm{CH}_{3}$ ), 2.92 (d, $\left.J=17.6 \mathrm{~Hz}, 1 \mathrm{H}, \mathrm{CHH}\right), 3.57$ (d, $J=17.6 \mathrm{~Hz}, 1 \mathrm{H}$, $\mathrm{CH} \mathrm{H}), 3.81\left(\mathrm{~s}, 3 \mathrm{H}, \mathrm{OCH}_{3}\right), 5.22\left(\mathrm{~s}, 2 \mathrm{H}, \mathrm{CH}_{2} \mathrm{ONO}_{2}\right) .{ }^{13} \mathrm{C} \mathrm{NMR}$ $\left(\mathrm{CDCl}_{3}, 75.47 \mathrm{MHz}, \mathrm{DEPT}\right): 23.2\left(\mathrm{CH}_{3}\right), 44.4\left(\mathrm{CH}_{2}\right), 53.1$ $\left(\mathrm{OCH}_{3}\right), 66.4\left(\mathrm{CH}_{2} \mathrm{ONO}_{2}\right), 86.8(C-\mathrm{O}), 151.7(C=\mathrm{N}), 171.6$ $(C=\mathrm{O}) .{ }^{14} \mathrm{~N} \mathrm{NMR}\left(\mathrm{CDCl}_{3}, 21.69 \mathrm{MHz}\right):-47.1$. FTIR (thin layer): $1647\left(\mathrm{~s}, \nu_{\text {as }} \mathrm{ONO}_{2}\right)$. HRMS: Calcd for $\left[\mathrm{C}_{7} \mathrm{H}_{10} \mathrm{~N}_{2} \mathrm{O}_{6} \mathrm{~K}^{+}\right]: \mathrm{m} / \mathrm{z}$ 257.0170 $\left([\mathrm{M}+\mathrm{K}]^{+}\right)$. Found: 257.0171.

[5-(4-Bromophenyl)-4,5-dihydroisoxazol-3-yl]methyl nitrate (2j). Yield: $65 \%$ (procedure i). White solid. Mp $49-51{ }^{\circ} \mathrm{C}$ (pentane/Et $\left.\mathrm{E}_{2} \mathrm{O}=5: 1\right) \cdot R_{\mathrm{f}}=0.7($ EtOAc/hexane $=1: 1) .{ }^{1} \mathrm{H}$ NMR $\left(\mathrm{CDCl}_{3}, 300.13 \mathrm{MHz}\right): 2.99$ (dd, $\left.J=17.3,8.5 \mathrm{~Hz}, 1 \mathrm{H}, \mathrm{HC}\right), 3.49$ (dd, $J=17.3,11.0 \mathrm{~Hz}, 1 \mathrm{H}, \mathrm{HC}$ ), 5.27 (s, $2 \mathrm{H}, \mathrm{CH}_{2}-\mathrm{ONO}_{2}$ ), 5.66 (dd, $J=11.0,8.5 \mathrm{~Hz}, 1 \mathrm{H}, H \mathrm{C}-\mathrm{Ar}), 7.20$ (d, $J=8.2 \mathrm{~Hz}, 2 \mathrm{H}$, $\left.\mathrm{C}_{6} \mathrm{H}_{4} \mathrm{Br}\right), 7.52$ (d, $\left.J=8.2 \mathrm{~Hz}, 2 \mathrm{H}, \mathrm{C}_{6} \mathrm{H}_{4} \mathrm{Br}\right) .{ }^{13} \mathrm{C} \mathrm{NMR}\left(\mathrm{CDCl}_{3}\right.$, $75.47 \mathrm{MHz}, \mathrm{DEPT}): 42.7\left(\mathrm{CH}_{2}\right), 66.6\left(\mathrm{CH}_{2}-\mathrm{ONO}_{2}\right), 82.4(\mathrm{CH})$, $122.5\left(p-C_{6} \mathrm{H}_{4} \mathrm{Br}\right), 127.4$ and $132.0\left(o-C_{6} \mathrm{H}_{4} \mathrm{Br}\right.$ and $\left.m-C_{6} \mathrm{H}_{4} \mathrm{Br}\right)$, $139.0(C-\mathrm{Br}), 151.4(C=\mathrm{N}) .{ }^{14} \mathrm{~N} \mathrm{NMR}\left(\mathrm{CDCl}_{3}, 21.69 \mathrm{MHz}\right)$ : -47.0. FTIR (KBr): 1640 (s, $\nu_{\text {as }} \mathrm{ONO}_{2}$ ), 525 (m, C-Br). Anal. Calcd for $\mathrm{C}_{10} \mathrm{H}_{9} \mathrm{BrN}_{2} \mathrm{O}_{4}$ : C, 39.89; $\mathrm{H}, 3.01 ; \mathrm{N}, 9.30$. Found: C, 39.71; H, 3.01; N, 9.11.

4-\{[5-(4-Bromophenyl)-4,5-dihydroisoxazol-3-yl $]$ methoxy\}butyl nitrate (6j). Yield: $18 \%$ (procedure i). Characterized in a mixture with $2 \mathbf{j}$ (ratio $6 \mathbf{j} / 2 \mathbf{j}=4.1: 1$ ). Oil. $R_{\mathrm{f}}=0.6($ EtOAc $/$ hexane $=$ 1:1). ${ }^{1} \mathrm{H} \mathrm{NMR}\left(\mathrm{CDCl}_{3}, 300.13 \mathrm{MHz}\right): 1.60-2.99(\mathrm{~m}, 4 \mathrm{H}$, $\left.\mathrm{CH}_{2}-\mathrm{CH}_{2}\right), 2.96(\mathrm{dd}, J=17.3,8.0 \mathrm{~Hz}, 1 \mathrm{H}, \mathrm{HC}), 3.46(\mathrm{dd}, J=$ 17.3, $10.5 \mathrm{~Hz}, 1 \mathrm{H}, \mathrm{HC}$ ), 3.47 (dd, $J=6.2,5.1 \mathrm{~Hz}, 2 \mathrm{H}, \mathrm{CH}_{2}-$ $\mathrm{CH}_{2}-\mathrm{O}$ ), 4.26 (s, $\left.2 \mathrm{H}, \mathrm{CH}_{2}-\mathrm{O}\right), 4.46(\mathrm{dd}, \mathrm{J}=6.2,6.2 \mathrm{~Hz}, 2 \mathrm{H}$, $\mathrm{CH}_{2}-\mathrm{ONO}_{2}$ ), 5.58 (dd, $\left.J=10.5,8.0 \mathrm{~Hz}, 1 \mathrm{H}, H \mathrm{C}-\mathrm{Ar}\right), 7.22$ (d, $J=$ $8.2 \mathrm{~Hz}, 2 \mathrm{H}, \mathrm{C}_{6} \mathrm{H}_{4} \mathrm{Br}$ ), 7.50 (d, $J=8.2 \mathrm{~Hz}, 2 \mathrm{H}, \mathrm{C}_{6} \mathrm{H}_{4} \mathrm{Br}$ ). ${ }^{13} \mathrm{C} \mathrm{NMR}\left(\mathrm{CDCl}_{3}, 75.47 \mathrm{MHz}, \mathrm{DEPT}\right): 23.8$ and $25.8\left(\mathrm{CH}_{2}-\mathrm{CH}_{2}\right)$, $43.4\left(\mathrm{CH}_{2}\right), 65.2,70.0$ and $73.0\left(\mathrm{CH}_{2}-\mathrm{O}, \mathrm{CH}_{2}-\mathrm{CH}_{2}-\mathrm{O}\right.$ and $\left.\mathrm{CH}_{2}-\mathrm{ONO}_{2}\right), 81.3(\mathrm{CH}), 122.1\left(p-C_{6} \mathrm{H}_{4} \mathrm{Br}\right), 127.4$ and 131.9 $\left(o-C_{6} \mathrm{H}_{4} \mathrm{Br}\right.$ and $\left.m-C_{6} \mathrm{H}_{4} \mathrm{Br}\right), 139.9(C-\mathrm{Br}), 156.0(C=\mathrm{N}) .{ }^{14} \mathrm{~N} \mathrm{NMR}$ $\left(\mathrm{CDCl}_{3}, 21.69 \mathrm{MHz}\right)$ : -41.4. IR: 1627 (s, $\left.\nu_{\text {as }} \mathrm{ONO}_{2}\right)$. HRMS: $\mathrm{m} / \mathrm{z}$ Calcd for $\left[\mathrm{C}_{14} \mathrm{H}_{17} \mathrm{BrN}_{2} \mathrm{O}_{5} \mathrm{Na}^{+}\right]$: 395.0213 and 397.0193 $\left([\mathrm{M}+\mathrm{Na}]^{+}\right)$. Found: 395.0216 and 397.0194. Contains diether $7 \mathbf{j}$ in trace amounts: Calcd for $\left[\mathrm{C}_{18} \mathrm{H}_{25} \mathrm{BrN}_{2} \mathrm{O}_{6} \mathrm{Na}^{+}\right]$: 467.0794 and $469.0776\left([\mathrm{M}+\mathrm{Na}]^{+}\right)$. Found: 467.0789 and 469.0775 .

rel-(4S,5S)-Ethyl 4-(4-methoxyphenyl)-3-(nitrooxymethyl)-4,5dihydroisoxazole-5-carboxylate (2k). Yield: 65\% (procedure ii). Oil. $R_{\mathrm{f}}=0.6$ (EtOAc/hexane $\left.=1: 1\right) .{ }^{1} \mathrm{H}$ NMR $\left(\mathrm{CDCl}_{3}\right.$, $300.13 \mathrm{MHz}$ ): 1.32 (t, $\left.J=7.1 \mathrm{~Hz}, 3 \mathrm{H}, \mathrm{OCH}_{2} \mathrm{CH}_{3}\right), 3.81(\mathrm{~s}, 3 \mathrm{H}$, $\left.\mathrm{OCH}_{3}\right), 4.29$ (q, $\left.J=7.1 \mathrm{~Hz}, 2 \mathrm{H}, \mathrm{OCH}_{2} \mathrm{CH}_{3}\right), 4.63(\mathrm{~d}, J=5.3 \mathrm{~Hz}$, $1 \mathrm{H}, \mathrm{CH}-4), 4.95$ (d, $\left.J=13.9 \mathrm{~Hz}, 1 \mathrm{H}, H \mathrm{C}-\mathrm{ONO}_{2}\right), 4.97(\mathrm{~d}, J=$ $5.3 \mathrm{~Hz}, 1 \mathrm{H}, \mathrm{CH}-5), 5.21$ (d, $J=13.9 \mathrm{~Hz}, 1 \mathrm{H}, \mathrm{HC}-\mathrm{ONO}_{2}$ ), 6.91 $\left(\mathrm{d}, J=8.6 \mathrm{~Hz}, 2 \mathrm{H}, o-\mathrm{C}_{6} \mathrm{H}_{4} \mathrm{OCH}_{3}\right), 7.12(\mathrm{~d}, J=8.6 \mathrm{~Hz}, 2 \mathrm{H}$, $\left.m-\mathrm{C}_{6} \mathrm{H}_{4} \mathrm{OCH}_{3}\right) .{ }^{13} \mathrm{C}$ NMR $\left(\mathrm{CDCl}_{3}, 75.47 \mathrm{MHz}, \mathrm{DEPT}\right): 14.0$ $\left(\mathrm{OCH}_{2} \mathrm{CH}_{3}\right), 55.4\left(\mathrm{OCH}_{3}\right), 57.1(\mathrm{CH}-4), 62.2$ and $64.8\left(2 \mathrm{CH}_{2}\right)$, $86.4(\mathrm{CH}-5), 115.0\left(o-C_{6} \mathrm{H}_{4} \mathrm{OCH}_{3}\right), 127.5\left(p-C_{6} \mathrm{H}_{4} \mathrm{OCH}_{3}\right), 128.7$ $\left(m-C_{6} \mathrm{H}_{4} \mathrm{OCH}_{3}\right), 154.1(C-\mathrm{O}), 159.9(C=\mathrm{N}), 169.9(C=\mathrm{O})$. ${ }^{14} \mathrm{~N}$ NMR $\left(\mathrm{CDCl}_{3}, 21.69 \mathrm{MHz}\right):-47.6$. FTIR (thin layer): 1741 (s, $\mathrm{C}=\mathrm{O}), 1650 \quad\left(\mathrm{~s}, \quad \nu_{\mathrm{as}} \mathrm{ONO}_{2}\right)$. HRMS: $\mathrm{m} / \mathrm{z}$ Calcd for $\left[\mathrm{C}_{14} \mathrm{H}_{17} \mathrm{~N}_{2} \mathrm{O}_{7}{ }^{+}\right] 325.1030\left([\mathrm{M}+\mathrm{H}]^{+}\right)$. Found: 325.1021. Anal. Calcd for $\mathrm{C}_{14} \mathrm{H}_{16} \mathrm{~N}_{2} \mathrm{O}_{7}$ : C, 51.85; H, 4.97; N, 8.64. Found: C, 51.86; H, 4.90; N, 8.60.

rel-(4S,5S)-Ethyl 3-(hydroxymethyl)-4-(4-methoxyphenyl)-4,5dihydroisoxazole-5-carboxylate (3k). Yield: 19\% (procedure ii). Isolated as a by-product to nitrate $2 \mathbf{k}$ by column chromatography. Characterized in a mixture with $\mathbf{6 k}$ (ratio $3 \mathbf{k} / \mathbf{6} \mathbf{k}=$ 7.6 : 1.0). Oil. $R_{\mathrm{f}}=0.5$ (EtOAc/hexane $\left.=1: 1\right) .{ }^{1} \mathrm{H}$ NMR $\left(\mathrm{CDCl}_{3}\right.$, $300.13 \mathrm{MHz}$ ): 1.30 (t, $\left.J=7.1 \mathrm{~Hz}, 3 \mathrm{H}, \mathrm{OCH}_{2} \mathrm{CH}_{3}\right), 2.76(\mathrm{br}, 1 \mathrm{H}$, 
$\mathrm{OH}), 3.79\left(\mathrm{~s}, 3 \mathrm{H}, \mathrm{OCH}_{3}\right), 4.11(\mathrm{~d}, J=14.3 \mathrm{~Hz}, 1 \mathrm{H}, \mathrm{CH}-\mathrm{OH})$, 4.25 (q, $J=7.1 \mathrm{~Hz}, 2 \mathrm{H}, \mathrm{OCH}_{2} \mathrm{CH}_{3}$ ), 4.37 (d, $J=14.3 \mathrm{~Hz}, 1 \mathrm{H}$, $\mathrm{CH}-\mathrm{OH}), 4.68$ (d, $J=5.2 \mathrm{~Hz}, 1 \mathrm{H}, \mathrm{CH}-4), 4.87$ (d, $J=5.2 \mathrm{~Hz}$, $1 \mathrm{H}, \mathrm{CH}-5), 6.89$ (d, $\left.J=8.6 \mathrm{~Hz}, 2 \mathrm{H}, o-\mathrm{C}_{6} H_{4} \mathrm{OCH}_{3}\right), 7.13(\mathrm{~d}, J=$ $\left.8.6 \mathrm{~Hz}, 2 \mathrm{H}, m-\mathrm{C}_{6} \mathrm{H}_{4} \mathrm{OCH}_{3}\right) .{ }^{13} \mathrm{C} \mathrm{NMR}\left(\mathrm{CDCl}_{3}, 75.47 \mathrm{MHz}\right.$, DEPT): $14.0\left(\mathrm{OCH}_{2} \mathrm{CH}_{3}\right), 55.3\left(\mathrm{OCH}_{3}\right), 57.5(\mathrm{CH}-4), 56.3$ and $62.0\left(2 \mathrm{CH}_{2}\right), 85.5(\mathrm{CH}-5), 114.8\left(o-C_{6} \mathrm{H}_{4} \mathrm{OCH}_{3}\right), 128.7$ $\left(m-C_{6} \mathrm{H}_{4} \mathrm{OCH}_{3}\right.$ and $\left.p-C_{6} \mathrm{H}_{4} \mathrm{OCH}_{3}\right), 159.6$ and $160.5(C-\mathrm{O}$ and $C=\mathrm{N}), 169.8(C=\mathrm{O})$. HRMS: $m / z$ Calcd for $\left[\mathrm{C}_{14} \mathrm{H}_{18} \mathrm{NO}_{5}{ }^{+}\right]$ $280.1179\left([\mathrm{M}+\mathrm{H}]^{+}\right)$. Found: 280.1179 .

rel-(4S,5S)-Ethyl 4-(4-methoxyphenyl)-3-((4-(nitrooxy)butoxy)methyl)-4,5-dihydroisoxazole-5-carboxylate (6k). Yield: 13\% (procedure ii). Isolated as a by-product to nitrate $\mathbf{2 k}$ by column chromatography. Characterized in a mixture with $2 \mathbf{k}$ (ratio 6k/2k = $2.5: 1)$. Oil. $R_{\mathrm{f}}=0.55($ EtOAc/hexane $=1: 1) .{ }^{1} \mathrm{H}$ NMR $\left(\mathrm{CDCl}_{3}, 300.13 \mathrm{MHz}\right): 1.35$ (t, $\left.J=7.1 \mathrm{~Hz}, 3 \mathrm{H}, \mathrm{OCH}_{2} \mathrm{CH}_{3}\right), 1.65$ and $1.77\left(2 \mathrm{~m}, 4 \mathrm{H}, \mathrm{CH}_{2}-\mathrm{CH}_{2}\right), 3.37$ and $3.48\left(2 \mathrm{~m}, 2 \mathrm{H}, \mathrm{CH}_{2}-\right.$ $\mathrm{CH}_{2}-\mathrm{O}$ ), 3.84 (s, $3 \mathrm{H}, \mathrm{OCH}_{3}$ ), 4.04 (d, $J=13.0 \mathrm{~Hz}, 1 \mathrm{H}, \mathrm{CH}_{2}-\mathrm{O}$ ), $4.20\left(\mathrm{~d}, J=13.0 \mathrm{~Hz}, 1 \mathrm{H}, \mathrm{CH}_{2}-\mathrm{O}\right), 4.31$ (q, $J=7.1 \mathrm{~Hz}, 2 \mathrm{H}$, $\left.\mathrm{OCH}_{2} \mathrm{CH}_{3}\right), 4.46$ (t, $\left.J=\mathrm{Hz}, 2 \mathrm{H}, \mathrm{CH}_{2}-\mathrm{CH}_{2}-\mathrm{ONO}_{2}\right), 4.64$ (d, $J=$ $5.0 \mathrm{~Hz}, 1 \mathrm{H}, \mathrm{CH}-4), 4.92(\mathrm{~d}, J=5.0 \mathrm{~Hz}, 1 \mathrm{H}, \mathrm{CH}-5), 6.93(\mathrm{~d}, J=$ $\left.8.6 \mathrm{~Hz}, 2 \mathrm{H}, o-\mathrm{C}_{6} \mathrm{H}_{4} \mathrm{OCH}_{3}\right), 7.16(\mathrm{~d}, J=8.6 \mathrm{~Hz}, 2 \mathrm{H}$, $\left.m-\mathrm{C}_{6} \mathrm{H}_{4} \mathrm{OCH}_{3}\right) .{ }^{13} \mathrm{C}$ NMR $\left(\mathrm{CDCl}_{3}, 75.47 \mathrm{MHz}\right.$, DEPT): 14.1 $\left(\mathrm{OCH}_{2} \mathrm{CH}_{3}\right), 23.7$ and $25.6\left(\mathrm{CH}_{2}-\mathrm{CH}_{2}\right), 55.3\left(\mathrm{OCH}_{3}\right), 57.6$ ( $\mathrm{CH}-4), 62.0,63.3,69.7$ and $73.0\left(4 \mathrm{CH}_{2}-\mathrm{O}\right), 85.4(\mathrm{CH}-5), 114.7$ $\left(o-C_{6} \mathrm{H}_{4} \mathrm{OCH}_{3}\right), 128.7\left(m-C_{6} \mathrm{H}_{4} \mathrm{OCH}_{3}\right.$ and $\left.p-C_{6} \mathrm{H}_{4} \mathrm{OCH}_{3}\right), 158.4$ $(C-\mathrm{O}), \quad 159.6 \quad(C=\mathrm{N}), \quad 169.7 \quad(C=\mathrm{O}) .{ }^{14} \mathrm{~N} \quad \mathrm{NMR} \quad\left(\mathrm{CDCl}_{3}\right.$, 21.69 MHz): -41.4. FTIR (thin layer): 1740 (s, sh, $\mathrm{C}=\mathrm{O}), 1649$ (s, sh, $\nu_{\text {as }} \mathrm{ONO}_{2}$ ). HRMS: $m / z$ Calcd for $\left[\mathrm{C}_{18} \mathrm{H}_{25} \mathrm{~N}_{2} \mathrm{O}_{8}{ }^{+}\right] 397.1605$ $\left([\mathrm{M}+\mathrm{H}]^{+}\right)$. Found: 397.1606 .

rel-((4S,6S)-4-(3-(Cyclopentyloxy)-4-methoxyphenyl)-6-((1S,2R)2-phenylcyclohexyloxy)-5,6-dihydro-4H-1,2-oxazin-3-yl)methyl nitrate (rac-2p). Yields: 46\% (procedure i), 35\% (procedure ii).

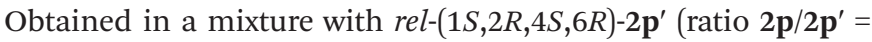
6.1:1 (procedure i), 5.8:1 (procedure ii), HPLC). Oil. Pure 2p can be obtained by crystallization from pentane $/ \mathrm{Et}_{2} \mathrm{O}$ mixtures $\left(\mathrm{Mp}=110-114^{\circ} \mathrm{C}\right) .{ }^{1} \mathrm{H}$ NMR $\left(\mathrm{CDCl}_{3}, 300 \mathrm{MHz}, \mathrm{COSY}, \mathrm{HSQC}\right)$ : 1.22-1.45, 1.49-1.75 and 1.77-2.10 (3 m, $17 \mathrm{H}, \mathrm{H}_{2} \mathrm{C}(5), \mathrm{H}_{2} \mathrm{C}(9)$, $H_{2} \mathrm{C}(10), H_{2} \mathrm{C}(11), H \mathrm{C}(12), H_{2} \mathrm{C}(22)$ and $\left.H_{2} \mathrm{C}(23)\right), 2.40(\mathrm{~m}, 1 \mathrm{H}$, $H \mathrm{C}(12)), 2.61$ (dd, $\left.J=11.2,10.7 \mathrm{~Hz}, 1 \mathrm{H}, H_{\mathrm{ax}} \mathrm{C}(8)\right), 2.90$ (dd, $J=$ 10.9, $\left.7.9 \mathrm{~Hz}, 1 \mathrm{H}, H_{\mathrm{ax}} \mathrm{C}(4)\right), 3.84$ (s, $\left.3 \mathrm{H}, \mathrm{H}_{3} \mathrm{C}(20)\right), 3.99$ (dd, $J=$ 10.7, $\left.9.9 \mathrm{~Hz}, 1 \mathrm{H}, H_{\mathrm{ax}} \mathrm{C}(7)\right), 4.22$ (d, $\left.J=13.3 \mathrm{~Hz}, 1 \mathrm{H}, H \mathrm{C}(13)\right)$, 4.39 (d, $J=13.3 \mathrm{~Hz}, 1 \mathrm{H}, H \mathrm{C}(13)), 4.73$ (br m, $1 \mathrm{H}, H \mathrm{C}(21)$ ), 5.41 (br s, $\left.1 \mathrm{H}, H_{\mathrm{eq}} \mathrm{C}(6)\right), 6.54$ (s, $\left.1 \mathrm{H}, H \mathrm{C}(15)\right), 6.58$ (d, $J=$ $7.1 \mathrm{~Hz}, 1 \mathrm{H}, H \mathrm{C}(19)), 6.79$ (d, $J=7.1 \mathrm{~Hz}, 1 \mathrm{H}, H \mathrm{C}(18))$, 7.16-7.39 (m, $\left.5 \mathrm{H}, \mathrm{C}_{6} \mathrm{H}_{5}\right) .{ }^{13} \mathrm{C} \mathrm{NMR}\left(\mathrm{CDCl}_{3}, 75.47 \mathrm{MHz}, \mathrm{HSQC}\right)$ : 24.0 and $24.6\left(\mathrm{CH}_{2}-9\right.$ and $\left.\mathrm{CH}_{2}-23\right), 26.1\left(\mathrm{CH}_{2}-10\right), 30.5\left(\mathrm{CH}_{2}-5\right)$, $31.8\left(\mathrm{CH}_{2}-12\right), 32.7$ and $32.8\left(\mathrm{CH}_{2}-22\right), 33.6(\mathrm{CH}-4), 34.0\left(\mathrm{CH}_{2}-\right.$ 11), 50.9 (CH-8), $56.1\left(\mathrm{CH}_{3}-20\right), 70.6\left(\mathrm{CH}_{2}-13\right), 76.3$ (CH-7), 80.6 (CH-21), 91.1 (CH-6), 112.4 (CH-18), 114.8 (CH-15), 120.6 $(\mathrm{CH}-19), 125.9\left(p-\mathrm{C}_{6} H_{5}\right), 127.9$ and $128.1\left(o-\right.$ and $\left.m-\mathrm{C}_{6} H_{5}\right)$, 130.1 (C-14), $144.5\left(\mathrm{i}-\mathrm{C}_{6} H_{5}\right), 148.2$ and 149.7 (C-16 and $\left.\mathrm{C}-17\right)$, $153.1(\mathrm{C}-3) .{ }^{14} \mathrm{~N}$ NMR $\left(\mathrm{CDCl}_{3}, 21.69 \mathrm{MHz}\right.$, rac-2p): -44.2. ${ }^{1} \mathrm{H}$ NMR spectra of rac-2p are in accordance with previously published data. ${ }^{1} \mathrm{H}$ NMR $\left(\mathrm{CDCl}_{3}, 300.13 \mathrm{MHz}\right.$, rac-2p', characteristic signals): $3.20\left(\mathrm{dd}, J=8.3,8.1 \mathrm{~Hz}, 1 \mathrm{H}, H_{\mathrm{eq}} \mathrm{C}-4\right), 3.84$ (s, $\left.3 \mathrm{H}, \mathrm{OCH}_{3}\right), 4.28\left(\mathrm{dd}, J=6.5,2.8 \mathrm{~Hz}, 1 \mathrm{H}, H_{\mathrm{eq}} \mathrm{C}-6\right), 4.68(\mathrm{~d}, J=$ $\left.12.9 \mathrm{~Hz}, 1 \mathrm{H}, \mathrm{HC}-\mathrm{ONO}_{2}\right), 4.80$ (d, $\left.J=12.9 \mathrm{~Hz}, 1 \mathrm{H}, H \mathrm{C}-\mathrm{ONO}_{2}\right)$.

\section{Reactions of model bis(oxy)enamine 1a with metal nitrates}

To a stirred solution of metal nitrate $(0.5 \mathrm{mmol})$ in $1 \mathrm{~mL}$ of solvent indicated in Table 1 was added $0.5 \mathrm{ml}$ of $0.5 \mathrm{M}$ solution of bis(oxy)enamine $(0.25 \mathrm{mmol})$ in $\mathrm{CH}_{2} \mathrm{Cl}_{2}$. The mixture was stirred at r.t. for the time indicated in Table 1 , and then poured into a mixture of EtOAc $(25 \mathrm{~mL})$ and $0.25 \mathrm{M} \mathrm{NaHSO}_{4}$ solution $(25 \mathrm{~mL})$. The aqueous layer was back-extracted with EtOAc $(25 \mathrm{~mL})$. Combined organic layers were washed with $0.25 \mathrm{M} \mathrm{NaHSO}_{4}$ solution $(25 \mathrm{~mL})$, water $(25 \mathrm{~mL})$, and brine $(25 \mathrm{~mL})$, dried $\left(\mathrm{Na}_{2} \mathrm{SO}_{4}\right)$, and evaporated in a vacuum. The residue was analyzed by ${ }^{1} \mathrm{H}$ NMR with an internal standard. ${ }^{1} \mathrm{H}$ NMR of by-product $3 \mathbf{a}$ is in accordance with published data. ${ }^{10 h}$ A sample of pure 4 a was prepared by standard silylation (r.t., $18 \mathrm{~h})$ of $3 \mathrm{a}(0.3 \mathrm{mmol})$ with $\left(\mathrm{CH}_{3}\right)_{3} \mathrm{SiCl}(0.6 \mathrm{mmol}) /$ $\mathrm{Et}_{3} \mathrm{~N}(0.75 \mathrm{mmol})$ in $\mathrm{CH}_{2} \mathrm{Cl}_{2}(1 \mathrm{~mL})$ followed by evaporation and extraction of the product with pentane (yield: 99\%). M.p. $=51-55{ }^{\circ} \mathrm{C} .{ }^{1} \mathrm{H}$ NMR $\left(\mathrm{CDCl}_{3}, 300.13 \mathrm{MHz}\right):-0.04$ $\left(\mathrm{s}, 9 \mathrm{H},\left(\mathrm{CH}_{3}\right)_{3} \mathrm{Si}\right), 1.33$ and $1.37\left(2 \mathrm{~s}, 6 \mathrm{H}, 2 \mathrm{CH}_{3}\right), 1.90$ (dd, $J=12.8,12.2 \mathrm{~Hz}, 1 \mathrm{H}, H \mathrm{C}-5), 2.11$ (dd, $J=12.8,8.1 \mathrm{~Hz}, 1 \mathrm{H}$, HC-5), 3.66 (dd, $J=12.2,8.1 \mathrm{~Hz}, 1 \mathrm{H}, H \mathrm{C}-4), 3.95(\mathrm{~d}, J=$ $11.7 \mathrm{~Hz}, 1 \mathrm{H}, \mathrm{CHO}$ ), 4.03 (d, $J=11.7 \mathrm{~Hz}, 1 \mathrm{H}, \mathrm{CHO}), 7.10-7.38$ (m, $\left.5 \mathrm{H}, o-, m-, p-\mathrm{C}_{6} H_{5}\right) .{ }^{13} \mathrm{C} \mathrm{NMR}\left(\mathrm{CDCl}_{3}, 75.47 \mathrm{MHz}, \mathrm{HSQC}\right)$ : $-0.37\left(\left(\mathrm{CH}_{3}\right)_{3} \mathrm{Si}\right), 22.9$ and $28.6\left(2 \mathrm{CH}_{3}\right), 36.9(\mathrm{CH}-4), 40.6$ $\left(\mathrm{CH}_{2}-5\right), 63.0\left(\mathrm{CH}_{2}-\mathrm{O}\right), 74.5(C-6), 127.1,128.6$ and 128.8 $\left(o-, m-, p-C_{6} \mathrm{H}_{5}\right), 140.5\left(\mathrm{i}-C_{6} \mathrm{H}_{5}\right), 158.0(C=\mathrm{N}) .{ }^{29} \mathrm{Si} \mathrm{NMR}\left(\mathrm{CDCl}_{3}\right.$, $59.63 \mathrm{MHz}):$ 19.9. HRMS: $m / z$ Calcd for $\left[\mathrm{C}_{16} \mathrm{H}_{26} \mathrm{NO}_{2} \mathrm{Si}^{+}\right]$ 292.1727 $\left([\mathrm{M}+\mathrm{H}]^{+}\right)$. Found: 292.1725 .

5-Hydroxy-2-(hydroxyimino)-5-methyl-3-phenylhexyl formate (5a). To a stirred solution of $\mathrm{Bi}\left(\mathrm{NO}_{3}\right)_{3} \cdot 5 \mathrm{H}_{2} \mathrm{O}(0.485 \mathrm{~g}$, $1.0 \mathrm{mmol}$ ) in DMF $(2.0 \mathrm{~mL})$ was added $1.0 \mathrm{~mL}$ of $0.5 \mathrm{M}$ solution of bis(oxy)enamine $\mathbf{1 a}(0.5 \mathrm{mmol})$ in $\mathrm{CH}_{2} \mathrm{Cl}_{2}$ at r.t. The mixture was stirred for $2.5 \mathrm{~h}$ at r.t. and then diluted with AcOEt $(5 \mathrm{~mL})$ and poured into a mixture of AcOEt $(50 \mathrm{~mL})$ and $0.25 \mathrm{M}$ aqueous solution of $\mathrm{NaHSO}_{4}(50 \mathrm{~mL})$. The aqueous layer was back-extracted with AcOEt $(50 \mathrm{~mL})$. Combined organic layers were washed with water $(30 \mathrm{~mL})$, and brine (30 $\mathrm{mL})$, dried $\left(\mathrm{Na}_{2} \mathrm{SO}_{4}\right)$, and evaporated in a vacuum. The residue was subjected to column chromatography on silica gel (eluent: hexane/AcOEt $=10: 1 \rightarrow 5: 1 \rightarrow 1: 1$ ) to give $0.045 \mathrm{~g}$ $(34 \%)$ of 5 a. Colorless oil. A mixture of $E, Z$-isomers, ratio $12: 1.0 . R_{\mathrm{f}}=0.38,0.32(2$ isomers, EtOAc/hexane $=1: 1)$. E-Isomer: ${ }^{1} \mathrm{H} \mathrm{NMR}\left(\mathrm{CDCl}_{3}, 300.13 \mathrm{MHz}\right)$ ): 1.26 and $1.30(2 \mathrm{~s}$, $6 \mathrm{H}, 2 \mathrm{CH}_{3}$ ), 1.84 (dd, $J=15.0,3.8 \mathrm{~Hz}, 1 \mathrm{H}, H \mathrm{C}-5$ ), 2.1 (br, $1 \mathrm{H}$, $\mathrm{OH}$ ), 2.48 (dd, $J=15.0,10.9 \mathrm{~Hz}, 1 \mathrm{H}, H \mathrm{C}-5), 3.98$ (dd, $J=10.9$, $3.8 \mathrm{~Hz}, 1 \mathrm{H}, H \mathrm{C}-4$ ), 4.58 (d, $J=14.6 \mathrm{~Hz}, 1 \mathrm{H}, H \mathrm{C}-\mathrm{O}$ ), 5.17 (d, $J=$ $14.6 \mathrm{~Hz}, 1 \mathrm{H}, \mathrm{HC}-\mathrm{O}), 7.12-7.38$ (m, $\left.5 \mathrm{H}, o-, m-, p-\mathrm{C}_{6} H_{5}\right), 7.91$ $(\mathrm{s}, 1 \mathrm{H}, H \mathrm{C}=\mathrm{O}), 10.7$ (br, $1 \mathrm{H}, \mathrm{NOH}) .{ }^{13} \mathrm{C} \mathrm{NMR}\left(\mathrm{CDCl}_{3}\right.$, $75.47 \mathrm{MHz}, \mathrm{DEPT}): 27.9$ and $31.5\left(2 \mathrm{CH}_{3}\right), 45.1(\mathrm{CH}-4), 47.3$ $\left(\mathrm{CH}_{2}-5\right), 57.4\left(\mathrm{CH}_{2} \mathrm{O}\right), 70.6(\mathrm{C}-6), 127.2,127.9$ and $129.2\left(o-, \mathrm{o}^{-}\right.$, $\left.p-C_{6} \mathrm{H}_{5}\right), 141.6\left(\mathrm{i}-C_{6} \mathrm{H}_{5}\right), 156.9(C=\mathrm{N}), 160.0(\mathrm{HC}=\mathrm{O}) . Z$-Isomer: $\left.{ }^{1} \mathrm{H} \mathrm{NMR} \mathrm{(} \mathrm{CDCl}_{3}, 300.13 \mathrm{MHz}\right): 1.39$ and $1.47\left(2 \mathrm{~s}, 6 \mathrm{H}, 2 \mathrm{CH}_{3}\right)$, 2.1 (br, $1 \mathrm{H}, \mathrm{OH}$ ), 2.14 (dd, $J=12.8,3.8 \mathrm{~Hz}, 1 \mathrm{H}, H \mathrm{C}-5), 2.58$ (dd, $J=12.8,12.6 \mathrm{~Hz}, 1 \mathrm{H}, H \mathrm{C}-5), 3.53$ (m, $1 \mathrm{H}, H \mathrm{C}-4), 4.76$ 
(d, $J=14.7 \mathrm{~Hz}, 1 \mathrm{H}, H \mathrm{C}-\mathrm{O}), 5.53$ (d, $J=14.7 \mathrm{~Hz}, 1 \mathrm{H}, H \mathrm{C}-\mathrm{O}$ ), 7.12-7.38 (m, $\left.5 \mathrm{H}, o-, m-, p-\mathrm{C}_{6} H_{5}\right), 7.91(\mathrm{~s}, 1 \mathrm{H}, H \mathrm{C}=\mathrm{O}), 10.7$ (br, $1 \mathrm{H}, \mathrm{OH}) .{ }^{13} \mathrm{C}$ NMR $\left(\mathrm{CDCl}_{3}, 75.47 \mathrm{MHz}, \mathrm{DEPT}\right.$, characteristic signals): 40.3 and $43.7\left(\mathrm{CH}-4\right.$ and $\left.\mathrm{CH}_{2}-5\right), 63.3\left(\mathrm{CH}_{2} \mathrm{O}\right)$. Both isomers: HRMS: $\mathrm{m} / \mathrm{z}$ Calcd for $\left[\mathrm{C}_{14} \mathrm{H}_{20} \mathrm{NO}_{4}{ }^{+}\right] 266.1387$ $\left([\mathrm{M}+\mathrm{H}]^{+}\right)$. Found: 266.1377 .

2-Phenyl-2-(trimethylsilyloxyimino)ethyl nitrate (2l) (procedure iii). To a stirred solution of anhydrous $\mathrm{Co}\left(\mathrm{NO}_{3}\right)_{2}(0.366 \mathrm{~g}$, $2.0 \mathrm{mmol})$ in THF $(4 \mathrm{~mL})$ was added $2 \mathrm{~mL}$ of $0.5 \mathrm{M}$ solution of bis(trimethysilyloxy)enamine $\mathbf{1 1}$. The resulting solution was stirred for $2 \mathrm{~h}$ at room temperature and poured into a mixture of EtOAc $(50 \mathrm{~mL})$ and saturated aqueous solution of $\mathrm{K}_{2} \mathrm{CO}_{3}$ $(50 \mathrm{~mL})$. The aqueous layer was back-extracted with EtOAc $(50 \mathrm{~mL})$. Combined organic layers were washed sequentially with saturated aqueous solution of $\mathrm{K}_{2} \mathrm{CO}_{3}(50 \mathrm{~mL})$, water $(50 \mathrm{~mL})$, and brine $(50 \mathrm{~mL})$, dried $\left(\mathrm{Na}_{2} \mathrm{SO}_{4}\right)$, and evaporated in a vacuum. The residue was dissolved in diethyl ether and filtered through a short column filled with charcoal $(0.5 \mathrm{~cm})$ and Celite $(0.5 \mathrm{~cm})$ layers to remove polymer products and traces of inorganic salts. The filtrate was evaporated to give $202 \mathrm{mg}$ $(65 \%)$ of oxime 21 as yellowish oil unstable at r.t. Purity ca. $85 \%$ (according to ${ }^{1} \mathrm{H}$ NMR with internal standard). A mixture of $Z / E$-isomers, ratio 9:1. $Z$-Isomer: ${ }^{1} \mathrm{H}$ NMR $\left(\mathrm{CDCl}_{3}\right.$, $300.13 \mathrm{MHz})$ : 0.35 (s, $\left.9 \mathrm{H},\left(\mathrm{CH}_{3}\right)_{3} \mathrm{Si}\right), 5.69$ (s, $2 \mathrm{H}, \mathrm{CH}_{2}-\mathrm{ONO}_{2}$ ), 7.39-7.46 and 7.63-7.67 $\left(2 \mathrm{~m}, 4 \mathrm{H}\right.$ and $\left.1 \mathrm{H}, o-, m-, p-\mathrm{C}_{6} H_{5}\right)$. ${ }^{13} \mathrm{C}$ NMR $\left(\mathrm{CDCl}_{3}, 75.47 \mathrm{MHz}, \mathrm{DEPT}\right):-0.76\left(\left(\mathrm{CH}_{3}\right)_{3} \mathrm{Si}\right), 64.3$ $\left(\mathrm{CH}_{2}-\mathrm{ONO}_{2}\right), 126.8,128.6$ and $129.9\left(o-, m-, p-C_{6} \mathrm{H}_{5}\right), 132.9$ $\left(\mathrm{i}-C_{6} \mathrm{H}_{5}\right), 154.8(\mathrm{C}=\mathrm{N}) .{ }^{29} \mathrm{Si} \mathrm{NMR}\left(\mathrm{CDCl}_{3}, 59.63 \mathrm{MHz}\right): 28.4$. ${ }^{14} \mathrm{~N}$ NMR $\left(\mathrm{CDCl}_{3}, 21.69 \mathrm{MHz}\right):-44.7$. E-Isomer: ${ }^{1} \mathrm{H}$ NMR $\left(\mathrm{CDCl}_{3}, 300.13 \mathrm{MHz}\right.$, characteristic signals): $0.34(\mathrm{~s}, 9 \mathrm{H}$, $\left.\left(\mathrm{CH}_{3}\right)_{3} \mathrm{Si}\right), 5.37\left(\mathrm{~s}, 2 \mathrm{H}, \mathrm{CH}_{2}-\mathrm{ONO}_{2}\right) \cdot{ }^{13} \mathrm{C} \mathrm{NMR}\left(\mathrm{CDCl}_{3}\right.$, $75.47 \mathrm{MHz}, \mathrm{DEPT}$, characteristic signals): $-0.64\left(\left(\mathrm{CH}_{3}\right)_{3} \mathrm{Si}\right)$, $62.2\left(\mathrm{CH}_{2}-\mathrm{ONO}_{2}\right)$. Both isomers: FTIR (thin layer): 1644 $\left(\mathrm{s}, \nu_{\text {as }} \mathrm{ONO}_{2}\right)$. MS (EI): $m / z(\%)=268$ (8) $[\mathrm{M}]^{+*}, 207$ (95) $\left[\mathrm{M}-\mathrm{CH}_{3}-\mathrm{NO}_{2}\right]^{+}, 192$ (73) $\left[\mathrm{M}-\mathrm{CH}_{2} \mathrm{ONO}_{2}\right]^{+}, 103$ (20) $\left[\mathrm{M}-\mathrm{Ph}-\mathrm{CH}_{2} \mathrm{ONO}_{2}\right]^{+}, 77(26)[\mathrm{Ph}]^{+}, 73(100)\left[\left(\mathrm{CH}_{3}\right)_{3} \mathrm{Si}\right]^{+}$.

\section{Synthesis of acyclic $\alpha$-nitroxyoximes 9m-o (procedure iv)}

To a stirred solution of anhydrous $\mathrm{Co}\left(\mathrm{NO}_{3}\right)_{2}(0.732 \mathrm{~g}$, $4.0 \mathrm{mmol})$ in a mixture of DMF $(4 \mathrm{~mL})$ and $\mathrm{CH}_{2} \mathrm{Cl}_{2}(4 \mathrm{~mL})$ was added $4 \mathrm{~mL}$ of $1 \mathrm{M}$ solution of bis(trimethysilyloxy)enamines $1 \mathbf{m}-\mathbf{o}(4.0 \mathrm{mmol})$ in $\mathrm{CH}_{2} \mathrm{Cl}_{2}$ at r.t. under an argon atmosphere. The mixture was stirred for $2.5 \mathrm{~h}$ at r.t., then poured into a mixture of AcOEt $(100 \mathrm{~mL})$ and $0.25 \mathrm{M}$ aqueous solution of $\mathrm{NaHSO}_{4}(100 \mathrm{~mL})$ and intensively shaken for ca. $5 \mathrm{~min}$. The aqueous layer was back-extracted with EtOAc $(50 \mathrm{~mL})$. The combined organic layers were washed with a $0.25 \mathrm{M}$ aqueous solution of $\mathrm{NaHSO}_{4}(70 \mathrm{~mL})$, water $(70 \mathrm{~mL})$, and brine $(70 \mathrm{~mL})$, dried $\left(\mathrm{Na}_{2} \mathrm{SO}_{4}\right)$, and concentrated in a vacuum. The residue was dissolved in $5 \mathrm{~mL}$ of $\mathrm{Et}_{2} \mathrm{O}$. Water $(1 \mathrm{~mL})$ was added and the mixture was intensively shaken for $1 \mathrm{~min}$. The organic layer was separated and the water phase was washed with $5 \mathrm{~mL}$ of $\mathrm{Et}_{2} \mathrm{O}$. Combined organic layers were dried over $\mathrm{Na}_{2} \mathrm{SO}_{4}$ and the volatiles were evaporated. The residue was dried in a vacuum (10 Torr) to give $\alpha$-nitroxyoximes 9m-o. Caution! Low molecular weight organic nitrates are explosive. No attempts to prepare more than $0.2 \mathrm{~g}$ were made.

2-(Hydroxyimino)ethyl nitrate (9m). A yellowish volatile liquid unstable upon heating and chromatography (purity ca. $90 \%$ determined by ${ }^{1} \mathrm{H}$ NMR with internal standard). Yield: $32 \%$ (with respect to purity). A mixture of $E / Z$-isomers, ratio 1.4 :1. E-Isomer: ${ }^{1} \mathrm{H}$ NMR $\left(\mathrm{CDCl}_{3}, 300.13 \mathrm{MHz}\right): 5.05$ (d, $J=$ $5.5 \mathrm{~Hz}, 2 \mathrm{H}, \mathrm{CH}_{2} \mathrm{ONO}_{2}$ ), $7.54(\mathrm{t}, J=5.5 \mathrm{~Hz}, 1 \mathrm{H},=\mathrm{CH}), 8.39$ (br, $1 \mathrm{H}, \mathrm{NOH}) \cdot{ }^{13} \mathrm{C} \mathrm{NMR}\left(\mathrm{CDCl}_{3}, 75.47 \mathrm{MHz}, \mathrm{DEPT}\right): 68.6$ $\left(\mathrm{CH}_{2} \mathrm{ONO}_{2}\right), 143.7(\mathrm{C}=\mathrm{N}) . \quad Z$-Isomer: ${ }^{1} \mathrm{H} \quad \mathrm{NMR} \quad\left(\mathrm{CDCl}_{3}\right.$, $300.13 \mathrm{MHz}): 5.29$ (d, $\left.J=3.7 \mathrm{~Hz}, 2 \mathrm{H}, \mathrm{CH}_{2} \mathrm{ONO}_{2}\right), 6.91(\mathrm{t}, J=$ $3.7 \mathrm{~Hz}, 1 \mathrm{H},=\mathrm{CH}), 8.74(\mathrm{br}, 1 \mathrm{H}, \mathrm{NOH}) .{ }^{13} \mathrm{C} \mathrm{NMR}\left(\mathrm{CDCl}_{3}\right.$, $75.47 \mathrm{MHz}$, DEPT): $65.8\left(\mathrm{CH}_{2} \mathrm{ONO}_{2}\right), 145.1(C=\mathrm{N})$. Both isomers: ${ }^{14} \mathrm{~N}$ NMR $\left(\mathrm{CDCl}_{3}, 21.69 \mathrm{MHz}\right)$ : -45.8. FTIR (thin layer): 3306 (s, br, OH), 2917 (m, CH), 1645 (s, $\nu_{\text {as }} \mathrm{ONO}_{2}$ ), $1426(\mathrm{~m}), 1277$ (s), 935 (m, sh), 852 (s). MS (EI): $\mathrm{m} / \mathrm{z}=$ $76\left[\mathrm{CH}_{2} \mathrm{ONO}_{2}\right]^{+}, 58\left[\mathrm{M}-\mathrm{NO}_{3}\right]^{+}, 57\left[\mathrm{M}-\mathrm{HNO}_{3}\right]^{+\bullet}, 46\left[\mathrm{NO}_{2}\right]^{+}$, $44\left[\mathrm{M}-\mathrm{CH}_{2} \mathrm{ONO}_{2}\right]^{+}$.

2-(Hydroxyimino)propyl nitrate (9n). A yellowish volatile liquid unstable upon heating and chromatography (purity ca. $80 \%$ determined by ${ }^{1} \mathrm{H}$ NMR with internal standard). Yield: $25 \%$ (with respect to purity). A mixture of $E / Z$-isomers, ratio 6.3 : 1. E-Isomer: ${ }^{1} \mathrm{H}$ NMR $\left(\mathrm{CDCl}_{3}, 300.13 \mathrm{MHz}\right): 1.98$ (s, $\left.\mathrm{CH}_{3}\right)$, $4.98\left(\mathrm{~s}, \mathrm{CH}_{2} \mathrm{ONO}_{2}\right), 9.12(\mathrm{br}, 1 \mathrm{H}, \mathrm{NOH}) .{ }^{13} \mathrm{C} \mathrm{NMR}\left(\mathrm{CDCl}_{3}\right.$, $75.47 \mathrm{MHz}, \mathrm{DEPT}): 12.0\left(\mathrm{CH}_{3}\right), 73.1\left(\mathrm{CH}_{2} \mathrm{ONO}_{2}\right), 151.2(\mathrm{C}=\mathrm{N})$. Z-Isomer: ${ }^{1} \mathrm{H}$ NMR $\left(\mathrm{CDCl}_{3}, 300.13 \mathrm{MHz}\right): 1.96$ (s, $\left.\mathrm{CH}_{3}\right), 5.33$ (s, $\mathrm{CH}_{2} \mathrm{ONO}_{2}$ ), 9.12 (br, $\left.1 \mathrm{H}, \mathrm{NOH}\right) .{ }^{13} \mathrm{C} \mathrm{NMR}\left(\mathrm{CDCl}_{3}, 75.47 \mathrm{MHz}\right.$, DEPT, characteristic signals): $15.7\left(\mathrm{CH}_{3}\right), 64.5\left(\mathrm{CH}_{2} \mathrm{ONO}_{2}\right)$. Both isomers: ${ }^{14} \mathrm{~N}$ NMR $\left(\mathrm{CDCl}_{3}, 21.69 \mathrm{MHz}\right)$ : -45.2. FTIR (thin layer): 3272 (s, br, OH), 2923 (m, CH), 1730 (m), 1644 $\left(\mathrm{s}, \nu_{\text {as }} \mathrm{ONO}_{2}\right), 1438(\mathrm{~m}), 1288(\mathrm{~s}), 945$ (m, sh), $921(\mathrm{~m}), 851(\mathrm{~s})$. MS (EI): $m / z=135[\mathrm{M}+\mathrm{H}]^{+}, 76\left[\mathrm{CH}_{2} \mathrm{ONO}_{2}\right]^{+}, 58\left[\mathrm{M}-\mathrm{NO}_{3}\right]^{+}$, $46\left[\mathrm{NO}_{2}\right]^{+}, 44\left[\mathrm{M}-\mathrm{CH}_{2} \mathrm{ONO}_{2}\right]^{+}$.

Methyl 4-(hydroxyimino)-5-(nitrooxy)pentanoate (9o). Yellowish oil unstable upon heating and chromatography (purity ca. $70 \%$ determined by ${ }^{1} \mathrm{H}$ NMR with internal standard). Yield: $27 \%$ (with respect to purity). Column chromatography (hexane/EtOAc $=5: 1$ ) on silica gel at $-15{ }^{\circ} \mathrm{C}$ provided a sample of individual 12c, which was used for further analysis. A mixture of $E / Z$-isomers, ratio $3.8: 1 . R_{\mathrm{f}}=0.55$ (EtOAc $/$ hexane $=$ 1:1). E-Isomer: ${ }^{1} \mathrm{H}$ NMR $\left(\mathrm{CDCl}_{3}, 300.13 \mathrm{MHz}\right): 2.50-2.70$ $\left(\mathrm{m}, 4 \mathrm{H}, \mathrm{CH}_{2}-\mathrm{CH}_{2}\right), 3.70\left(\mathrm{~s}, 3 \mathrm{H}, \mathrm{OCH}_{3}\right), 5.07(\mathrm{~s}, 2 \mathrm{H}$, $\left.\mathrm{CH}_{2} \mathrm{ONO}_{2}\right), 9.18$ (br, $\left.1 \mathrm{H}, \mathrm{NOH}\right) .{ }^{13} \mathrm{C} \mathrm{NMR}\left(\mathrm{CDCl}_{3}, 75.47 \mathrm{MHz}\right.$, DEPT): 22.0 and $29.7\left(\mathrm{CH}_{2}-\mathrm{CH}_{2}\right), \quad 52.0 \quad\left(\mathrm{OCH}_{3}\right), \quad 72.5$ $\left(\mathrm{CH}_{2} \mathrm{ONO}_{2}\right), 153.0(\mathrm{C}=\mathrm{N}), 173.2(C=\mathrm{O}) . Z$-Isomer: ${ }^{1} \mathrm{H}$ NMR $\left(\mathrm{CDCl}_{3}, \quad 300.13 \mathrm{MHz}\right): \quad 2.50-2.70$ (m, $\left.4 \mathrm{H}, \mathrm{CH}_{2}-\mathrm{CH}_{2}\right)$, $3.80\left(\mathrm{~s}, 3 \mathrm{H}, \mathrm{OCH}_{3}\right), 5.33\left(\mathrm{~d}, J=3.7 \mathrm{~Hz}, 2 \mathrm{H}, \mathrm{CH}_{2} \mathrm{ONO}_{2}\right.$ ), 9.03 (br, $1 \mathrm{H}, \mathrm{NOH}) .{ }^{13} \mathrm{C} \mathrm{NMR}\left(\mathrm{CDCl}_{3}, 75.47 \mathrm{MHz}, \mathrm{DEPT}\right)$ : 25.7 and $29.9\left(\mathrm{CH}_{2}-\mathrm{CH}_{2}\right), 52.0\left(\mathrm{OCH}_{3}\right), 66.8\left(\mathrm{CH}_{2} \mathrm{ONO}_{2}\right)$, $155.1(\mathrm{C}=\mathrm{N}), \quad 173.9 \quad(\mathrm{C}=\mathrm{O})$. Both isomers: ${ }^{14} \mathrm{~N} \quad \mathrm{NMR}$ $\left(\mathrm{CDCl}_{3}, 21.69 \mathrm{MHz}\right):-45.2$. FTIR (thin layer): 3382 (s, br, $\mathrm{OH}$ ), 2957 (m, sh, CH), 1733 (s, C=O), 1644 (s, $\nu_{\text {as }} \mathrm{ONO}_{2}$ ), 1440 (m), 1368 (m), 1283 (s, sh), 1203 (m), 1176 (m), 983 (m, sh), 851 (s), $640(\mathrm{w})$. HRMS: $m / z$ Calcd for $\left[\mathrm{C}_{6} \mathrm{H}_{10} \mathrm{~N}_{2} \mathrm{O}_{6} \mathrm{Na}^{+}\right]$ $229.0431\left([\mathrm{M}+\mathrm{Na}]^{+}\right)$. Found: 229.0434. MS (EI): $\mathrm{m} / \mathrm{z}=$ $206[\mathrm{M}]^{+}$. 


\section{Acknowledgements}

This work was supported by the Russian Science Foundation (Grant 14-50-00126).

\section{Notes and references}

1 (a) A. Koenig, K. Lange, J. Konter, A. Daiber, D. Stalleicken, E. Glusa and J. Lehmann, J. Cardiovasc. Pharmacol., 2007, 50, 68-74; (b) K. Lange, A. Koenig, C. Roegler, A. Seeling and J. Lehmann, Bioorg. Med. Chem. Lett., 2009, 19, 31413144; (c) M. W. Dewhirst, J. S. Stamler, T. J. Mcmahon and P. Sonveaux, Patent WO 2006/113540A2, 2006 (Duke University); (d) I. V. Serkov and V. V. Bezuglov, Russ. Chem. Rev., 2009, 78, 407-429; (e) P. L. Bosquesi, T. R. F. Melo, E. O. Vizioli, J. Leandro dos Santos and M. C. Chung, Pharmaceuticals, 2011, 4, 1450-1474; (f) S. G. Zlotin, A. M. Churakov, O. A. Luk'yanov, N. N. Makhova, A. Yu. Sukhorukov and V. A. Tartakovsky, Mendeleev Commun., 2015, 25, 399-409; $(g)$ L. L. Fershtat, M. A. Epishina, A. S. Kulikov, I. V. Ovchinnikov, I. V. Ananyev and N. N. Makhova, Tetrahedron, 2015, 71, 6764-6775; (h) G. A. Smirnov, P. B. Gordeev, S. V. Nikitin, G. V. Pokhvisneva, T. V. Ternikova and O. A. Luk'yanov, Russ. Chem. Bull., Int. Ed., 2015, 64, 1057-1061.

2 (a) A. K. Shaikh, A. J. A. Cobb and G. Varvounis, Org. Lett., 2012, 14, 584-587; (b) A. K. Shaikh and G. Varvounis, Org. Lett., 2014, 16, 1478-1481; (c) T. H. Graham, C. M. Jones, N. T. Jui and D. W. C. MacMillan, J. Am. Chem. Soc., 2008, 130, 16494-16495.

3 (a) R. Caderas, R. Lett, L. Overman, M. H. Rabinowitz, L. A. Robinson, M. Z. Sharp and J. Zablocki, J. Am. Chem. Soc., 1996, 118, 9073-9082; (b) Z. Qin, J. Luo, L. Vandevrede, E. Tavassoli, M. Fa, A. F. Teich, O. Arancio and G. R. J. Thatcher, J. Med. Chem., 2012, 55, 6784-6801.

4 (a) N. Kornblum and H. W. Frazier, J. Am. Chem. Soc., 1966, 88, 865; (b) D. L. Lindner, J. B. Doherty, G. Shoham and R. B. Woodward, Tetrahedron Lett., 1982, 23, 5111-5114; (c) M. J. Melnick and S. M. Weinreb, J. Org. Chem., 1988, 53, 850-854.

5 (a) J. Prakash Agrawal, High Energy Materials: Propellants, Explosives and Pyrotechnics, Wiley-VCH, Weinheim, 2010; (b) D. E. Chavez, M. A. Hiskey, D. L. Naud and D. Parrish, Angew. Chem., Int. Ed., 2008, 47, 8307-8309.

6 G. A. Olah, R. Malhotra and S. C. Narang, Nitration. Methods and Mechanisms (Organic Nitro Chemistry Series), VCH Verlagsgesellschaft, Weinheim, VCH Publishers, New York, 1989.

7 (a) A. F. Ferris, K. W. McLean, I. G. Marks and W. D. Emmons, J. Am. Chem. Soc., 1953, 75, 4078-4078; (b) D. Garvey, L. Letts, R. Earl, M. Ezawa, X. Fang, R. Gaston, S. Khanapure, C.-E. Lin, R. Ranatunge, C. Stevenson and S.-J. Wey, Patent US 20060189603A1, 2006 (Nitromed, Inc.); (c) L. Lazzarato, M. Donnola, B. Rolando, K. Chegaev, E. Marini, C. Cena, A. Di Stilo, R. Fruttero,
S. Biondi, E. Ongini and A. Gasco, J. Med. Chem., 2009, 52, 5058-5068; (d) A. McKillop and M. E. Ford, Tetrahedron, 1974, 30, 2467-2475.

8 (a) X.-T. Chen, D. Sames and S. J. Danishefsky, J. Am. Chem. Soc., 1998, 120, 7760-7769; (b) S. Lee and J. N. P. Rosazza, Org. Lett., 2004, 6, 365-368; (c) E. Baciocchi, C. Rol, G. V. Sebastiani and A. Zampini, J. Chem. Soc., Chem. Commun., 1982, 1045-1047; (d) M.-Y. Chang, C.-Y. Lin and T.-C. Wu, Tetrahedron Lett., 2006, 47, 5445-5449; (e) V. Nair, S. B. Panicker, A. Augustine, T. G. George, S. Thomas and M. Vairamani, Tetrahedron, 2001, 57, 7417-7422.

9 (a) A. Michael and G. H. Carlson, J. Am. Chem. Soc., 1935, 57, 1268-1276; (b) I. Dunstan, J. V. Griffiths and S. A. Harvey, J. Chem. Soc., 1965, 1319-1324; (c) J. Barluenga, J.-M. Martinez-Gallo, C. Najera and M. Yus, J. Chem. Soc., Chem. Commun., 1985, 20, 1422-1423; (d) R. J. Lewis and R. B. Moodie, J. Chem. Soc., Perkin Trans. 2, 1996, 1315-1320; (e) H. Wieland and F. Rahn, Berichte, 1921, 54, 1770-1776; (f) A. A. Stotskii, V. V. Kirichenko and L. I. Bagal, J. Org. Chem. USSR, 1973, 9, 2486-2491.

10 (a) A. A. Tishkov, A. D. Dilman, V. I. Faustov, A. A. Birukov, K. S. Lysenko, P. A. Belyakov, S. L. Ioffe, Y. A. Strelenko and M. Yu. Antipin, J. Am. Chem. Soc., 2002, 124, 11358-11367; (b) H. Feger and G. Simchen, Liebigs Ann. Chem., 1986, 1456-1465; (c) A. A. Tishkov, A. V. Lesiv, Yu. A. Khomutova, Yu. A. Strelenko, I. D. Nesterov, M. Yu. Antipin, S. L. Ioffe and S. E. Denmark, J. Org. Chem., 2003, 68, 9477-9480; (d) A. D. Dilman, A. A. Tishkov, I. M. Lyapkalo, S. L. Ioffe, Yu. A. Strelenko and V. A. Tartakovsky, Synthesis, 1998, 181-184; (e) A. D. Dilman, S. L. Ioffe and H. Mayr, J. Org. Chem., 2001, 66, 3196-3200; (f) M. S. Klenov, A. V. Lesiv, Yu. A. Khomutova, I. D. Nesterov and S. L. Ioffe, Synthesis, 2004, 1159-1170; (g) A. A. Tabolin, A. V. Lesiv, Yu. A. Khomutova, Yu. V. Nelyubina and S. L. Ioffe, Tetrahedron, 2009, 65, 4578-4592; (h) A. A. Tabolin, A. V. Lesiv and S. L. Ioffe, Synthesis, 2009, 3099-3105; (i) A. A. Mikhaylov, A. D. Dilman, M. I. Struchkova, Yu. A. Khomutova, A. A. Korlyukov, S. L. Ioffe and V. A. Tartakovsky, Tetrahedron, 2011, 67, 4584-4594; (j) A. A. Mikhaylov, P. A. Zhmurov, A. S. Naumova, Y. A. Khoroshutina, A. Yu. Sukhorukov and S. L. Ioffe, Mendeleev Commun., 2015, 25, 449-451.

11 (a) S. L. Ioffe, in Nitrile Oxides, Nitrones, and Nitronates in Organic Synthesis: Novel Strategies in Synthesis, ed. H. Feuer, Wiley, Hoboken, 2nd edn, 2008, p. 658; (b) A. Yu. Sukhorukov and S. L. Ioffe, Chem. Rev., 2011, 111, 5004-5041; (c) A. A. Tabolin and S. L. Ioffe, Chem. Rev., 2014, 114, 5426-5476. For a related process see: (d) T. Miyoshi, T. Miyakawa, M. Ueda and O. Miyata, Angew. Chem., Int. Ed., 2011, 123, 958-961.

12 Only one example of acyclic $\alpha$-nitroxy-oxime has been authentically reported: O. A. Luk'yanov and G. V. Pokhvisneva, Bull. Acad. Sci. USSR, Div. Chem. Sci., 1991, 40, 2439-2444. Only one example of 5 -membered cyclic $\alpha$-nitroxy-oxime ether has been obtained as a by-product: J. F. Hansen and P. J. Georgiou, J. Heterocycl. Chem., 1994, 31, 1487-1492. 
13 (a) F. Guthrie, Ann. Chem. Pharm., 1860, 116, 234-249; (b) O. Wallach, Ann. Chem., 1887, 241, 288-315; (c) V. N. Ipatiew and A. A. Solonina, Zh. Russ. Fiz.-Khim. O-va., 1901, 33, 496-501.

14 A. Yu. Sukhorukov, M. A. Kapatsyna, T. Lim Ting Yi, H. Park, Ya. A. Naumovich, P. A. Zhmurov, Yu. A. Khomutova, S. L. Ioffe and V. A. Tartakovsky, Eur. J. Org. Chem., 2014, 8148-8159.

15 For processes involving $N$-alkoxynitrenium cations as intermediates see: (a) V. F. Rudchenko, Chem. Rev., 1993, 93, 725-739; (b) J. J. Campbell, S. A. Glover, G. P. Hammond and C. A. Rowbottom, J. Chem. Soc., Perkin Trans. 2, 1991, 2067-2079; (c) V. O. Smirnov, Yu. A. Khomutova, V. A. Tartakovsky and S. L. Ioffe, Eur. J. Org. Chem., 2012, 3377-3384; (d) A. A. Tabolin, A. V. Lesiv, Yu. A. Khomutova, P. A. Belyakov, Yu. A. Strelenko and S. L. Ioffe, Synthesis, 2005, 1656-1662.
16 For some other applications of the $\mathrm{ONO}_{2}$ group in total synthesis see: (a) P. W. Glunz, S. Hintermann, L. J. Williams, J. B. Schwarz, S. D. Kuduk, V. Kudryashov, K. O. Lloyd and S. J. Danishefsky, J. Am. Chem. Soc., 2000, 122, 7273-7279; (b) T. K. Jones, H. Mrozik and M. H. Fisher, J. Org. Chem., 1992, 57, 3248-3250; (c) A. L. Parry, N. A. Clemson, J. Ellis, S. S. R. Bernhard, B. G. Davis and N. R. Cameron, J. Am. Chem. Soc., 2013, 135, 9362-9365.

17 (a) P. A. Zhmurov, A. Yu. Sukhorukov, V. I. Chupakhin, Yu. A. Khomutova, S. L. Ioffe and V. A. Tartakovsky, Org. Biomol. Chem., 2013, 11, 8082-8091; (b) A. Yu. Sukhorukov, Y. D. Boyko, Yu. A. Khomutova, Yu. V. Nelyubina, S. L. Ioffe and V. A. Tartakovsky, J. Org. Chem., 2011, 76, 7893-7900.

18 C. Ehrhardt, M. Gjikaj and W. Brockner, Thermochim. Acta, 2005, 432, 36-40.

19 H. Wong, P. Hanselmann and W. Wenger, Patent WO 2010/ 45764A1, 2010 (Lonza Ltd). 